\title{
ESTRUCTURA DEL BOSQUE MESÓFILO DE MONTAÑA Y SU REEMPLAZO POR BOSQUE DE ABIES EN DOS GRADIENTES ALTITUDINALES DEL OCCIDENTE DE MÉXICO
}

\section{CLOUD FOREST STRUCTURE AND ITS REPLACEMENT BY FIR FOREST ALONG TWO ALTITUDINAL GRADIENTS IN WESTERN MEXICO}

\author{
Ricardo Guerrero-Hernández ${ }^{1}$, Miguel Ángel MuñIz-Castro ${ }^{1 *}$, J. Antonio Vázquez-García ${ }^{1}$ y J. Ariel \\ RUIZ-CORRAL ${ }^{2}$
}

\author{
${ }^{1}$ Instituto de Botánica, Departamento de Botánica y Zoología, Centro Universitario de Ciencias Biológicas y Agropecuarias, \\ Universidad de Guadalajara, Zapopan, Jalisco, México. \\ ${ }^{2}$ Campo Experimental Centro Altos Jalisco, Instituto Nacional de Investigaciones Forestales, Tepatitlán de Morelos, Jalisco, \\ México.
}

*Autor para correspondencia: miguel.muniz@academicos.udg.mx

\begin{abstract}
Resumen
Antecedentes: El occidente de Jalisco alberga archipiélagos de bosque mesófilo de montaña, los cuales, al igual que en el este de México, tienen una alta riqueza de especies arbóreas raras y con distribución restringida. En su límite superior altitudinal forman ecotonos con bosques de Abies.

Hipótesis: Con el incremento de la altitud, hay un cambio direccional en la distribución de las especies dominantes, estructura de la vegetación y disminución en riqueza de especies leñosas.

Descripción de datos: Los datos analizados incluyeron área basal, altura, abundancia, diversidad Shannon, riqueza y variables ambientales. Sitio de estudio y año de estudio: El occidente de Jalisco, en dos localidades con bosque mesófilo de montaña y bosque de Abies, desde septiembre de 2013 hasta agosto de 2015.

Métodos: Se midieron e identificaron especies leñosas en 32 unidades de muestreo de 0.1 ha; mediante análisis de regresión y métodos multivariados se exploraron variables ambientales asociadas a grupos de vegetación en relación con la altitud.

Resultados: Se detectaron patrones unimodales y lineales de estructura y diversidad en ambos gradientes. Los análisis sugieren cinco grupos: bosque mesófilo de baja montaña asociado a temperatura media anual, precipitación anual y pedregosidad; bosque mesófilo de alta montaña y bosque de coníferas (Abies) asociados a la altitud y precipitación invernal; y dos grupos dominados por Quercus-Pinus.

Conclusiones: Los resultados muestran que distintas especies dominantes están asociadas a diferentes condiciones climáticas y sugieren patrones distintos en cada localidad: a) la riqueza disminuye con la altitud en La Bufa; b) la riqueza y la estructura muestran un patrón unimodal en Laguna Juanacatlán.
\end{abstract}

Palabras clave: Bosque de Abies, bosque mesófilo de montaña, especies indicadoras, gradiente altitudinal, precipitación, temperatura.

\begin{abstract}
Background: Western Jalisco hosts archipelagos of Tropical Montane Cloud Forest, which have a high richness of rare tree species with restricted distribution same as in the eastern Mexico. At its upper limit of elevation form ecotones with fir forests.

Hypotheses: With the increase in elevation, there is a directional change in the distribution of dominant species, vegetation structure and a decrease in woody species richness.

Data description: The data analyzed included basal area, stems height, abundance, Shannon diversity, richness, and environmental variables.

Study site and dates: This study was conducted in western Jalisco, at two localities with Tropical Montane Cloud Forest and Fir Forest, from September 2013 to August 2015.

Methods: Woody species were identified and measured in 32 sampling units of 0.1 ha; regression analysis and multivariate methods were used to explore environmental variables associated with vegetation groups in relation to altitude.

Results: Unimodal and linear patterns in structure and diversity were detected in both gradients. The analysis indicates five groups: lower montane cloud forest associated to mean annual temperature, annual precipitation and stoniness; upper montane cloud forest and fir forest associated to elevation and winter precipitation; and two groups dominated by Quercus-Pinus.

Conclusions: The results show that different dominant species are associated with different climatic conditions and support two patterns depending of locality: a) Species richness decreases linearly with elevation in La Bufa; b) Species richness and structure show a unimodal pattern in Laguna Juanacatlán.
\end{abstract}

Keywords: Altitudinal gradient, cloud forest, fir forest, indicator species, precipitation, temperature.

This is an open access article distributed under the terms of the Creative Commons Attribution License CCBY-NC (4.0) international. https://creativecommons.org/licences/by-nc/4.0/ 
En los ecosistemas de montaña, la altitud, el microambiente y el clima juegan un rol importante en la distribución y estructura de las comunidades vegetales. Particularmente, el bosque mesófilo de montaña (BMM) (Rzedowski 1978), conocido también como bosque de niebla en México, y en inglés como "Tropical Montane Cloud Forest" (Hamilton et al. 1995), el cual alberga elementos de distinta afinidad biogeográfica, representa una gran oportunidad para evaluar las relaciones entre microambiente, clima, cambios en la estructura de la vegetación y su composición florística. Esta última ya ha sido documentada previamente a nivel mundial (Vázquez-García 1995, Hamilton et al. 1995, Bruijnzeel et al. 2010) y en México-Centroamérica (Lieberman et al. 1996, Alcántara-Ayala \& Luna-Vega 1997, Catalán-Heverástico et al. 2003, CONABIO 2010, Villaseñor 2010, Williams-Linera et al. 2013). La riqueza florística y rasgos estructurales de los bosques de niebla han sido relacionados con la altitud, y se han encontrado tendencias contrastantes, tales como unimodales, de disminución o aumento lineal monotónico (Tang \& Ohsawa 1997, Rahbek 2005, Homeier et al. 2010, Salas-Morales \& Meave 2012, Krömer et al. 2013, Williams-Linera et al. 2013). Estas tendencias varían entre regiones y entre las escalas consideradas para el análisis de los intervalos altitudinales, es decir, también han sido encontradas en áreas extensas donde se estudió toda la vegetación en transectos con presencia de bosques tropicales caducifolios hasta bosques subalpinos, y no solamente el BMM (Kitayama 1992, Vázquez-García \& Givnish 1998, Reich et al. 2010, Hemp 2010, Toledo-Garibaldi \& Williams-Linera 2014).

En el sureste de Asia algunos investigadores han separado los bosques de niebla (el término BMM solo es utilizado en México) en bosques de niebla de baja y alta montaña (lower montane cloud forest y upper montane cloud forest) y bosques de niebla dominados por coníferas (Ohsawa 1991, 1995), en relación con la franja latitudinal y altitudinal en la que estén distribuidos. Además, varios autores han tratado de distinguir límites discretos (Grubb 1977, Hemp 2010, Martin et al. 2011, Bach \& Gradstein 2011), aunque otros argumentan que los cambios estructurales y en composición de especies ocurren continuamente a través del gradiente (Lieberman et al. 1996). En contraste, en México no se ha aceptado el enfoque de separar el BMM en fajas altitudinales distintas, aunque en algunos estudios se han reconocido subtipos de BMM y distintas especies dominantes (Alcántara et al. 2002, Mejía-Domínguez et al. 2004, Williams-Linera et al. 2013). Un ejemplo de los distintos ensambles, componentes dominantes y subtipos de BMM son las ecotonías en su límite superior de distribución altitudinal que forman con los bosques de Abies (abeto, oyamel o pinabete). Estas ecotonías suelen presentarse en el occidente y sur de México, incluso hasta Guatemala (Ohsawa 1995, Cuevas-Guzmán et al. 2011, Guerrero-Hernández et al. 2014); sin embargo, ninguna investigación ha examinado específicamente la transición entre BMM y el bosque de Abies, y la relación de ambas comunidades vegetales con variables microambientales y climáticas a lo largo de gradientes altitudinales.

La porción occidental de Jalisco alberga archipiélagos de bosque mesófilo de montaña, los cuales, al igual que en la vertiente del Golfo de México, tienen una alta riqueza de especies arbóreas raras y con distribución restringida (Vázquez-García 1995, Jardel-Pelaez et al. 1996, Vázquez-García et al. 2000, Sánchez-Rodríguez et al. 2003, Guerrero-Hernández et al. 2014). A pesar de esto, la mayoría de estos bosques no figuran entre los hotspots de biodiversidad en la WWF, excepto Sierra de Manantlán (Cuevas-Guzmán et al. 1997). Por otro lado, probablemente los bosques de Abies ocupan mayores extensiones que los BMM, pero son relictos de una distribución que fue aún más amplia durante el Mioceno tardío (Graham 1999), y en la actualidad se distribuyen mayormente en zonas altas y frías de la Faja Volcánica Trans-Mexicana. Al igual que otros taxa de origen templado, el género Abies ha estado expuesto a una dinámica de "islas en el cielo" como respuesta a fluctuaciones climáticas (Mastretta-Yanes et al. 2015). Dada la distribución insular y restringida que exhiben estas comunidades vegetales, es necesario generar información de la estructura y dinámica vegetal para implementar estrategias de manejo y conservación tanto de los BMM como de los bosques de Abies en el occidente de México.

En esta investigación se exploran tendencias en composición de especies leñosas, cambios en la estructura de la vegetación, microambiente y clima en relación con la altitud en dos localidades del occidente de México. Las hipótesis que se plantearon son que en el área de estudio (1) con el incremento de la altitud, hay un cambio progresivo y continuo en la distribución de las especies dominantes, estructura de la vegetación y una disminución monotónica de la riqueza de especies leñosas; (2) el reemplazo del bosque mesófilo de montaña por bosque de oyamel está positivamente asociado con la altitud, disminución de temperatura y mayor disponibilidad de agua en los meses más fríos y secos del año. Nuestros objetivos específicos fueron (1) analizar, en dos gradientes altitudinales, cambios en la estructura de la vegetación y composición de especies leñosas, con base en área basal, altura, abundancia y riqueza; (2) examinar las relaciones entre variables climáticas, factores microambientales y la estructura de la vegetación.

\section{Materiales y métodos}

Área de estudio. El área de estudio comprende dos localidades con bosque mesófilo de montaña y bosque de Abies, que se encuentran en la Sierra de Jolapa, en el occidente del estado de Jalisco, México (Figura 1). Se delimita entre las coordenadas $20^{\circ} 45^{\prime} 15^{\prime \prime}$ y $20^{\circ} 38^{\prime} 07^{\prime \prime} \mathrm{N}$ y $104^{\circ} 50^{\prime} 29^{\prime}$ " y $104^{\circ} 42^{\prime} 47^{\prime \prime}$ O (DATUM WGS 84), en los municipios de Mascota y San Sebastián del Oeste. Se registraron dos intervalos altitudinales, el primero abarca de los 1,500 a los 2,450 m en la localidad de La Bufa (B), y el segundo de los 1,750 a los 2,420 m en la localidad de Laguna Juanacatlán (J). La mayor parte de esta superficie está localizada dentro del Área de Protección de Recursos Naturales Río AmecaSierra de Vallejo. Se ubica en la cuenca hidrológica AmecaIxtapa, en la provincia morfotectónica de la Faja Volcánica Trans-Mexicana, en una zona donde converge con algunos 

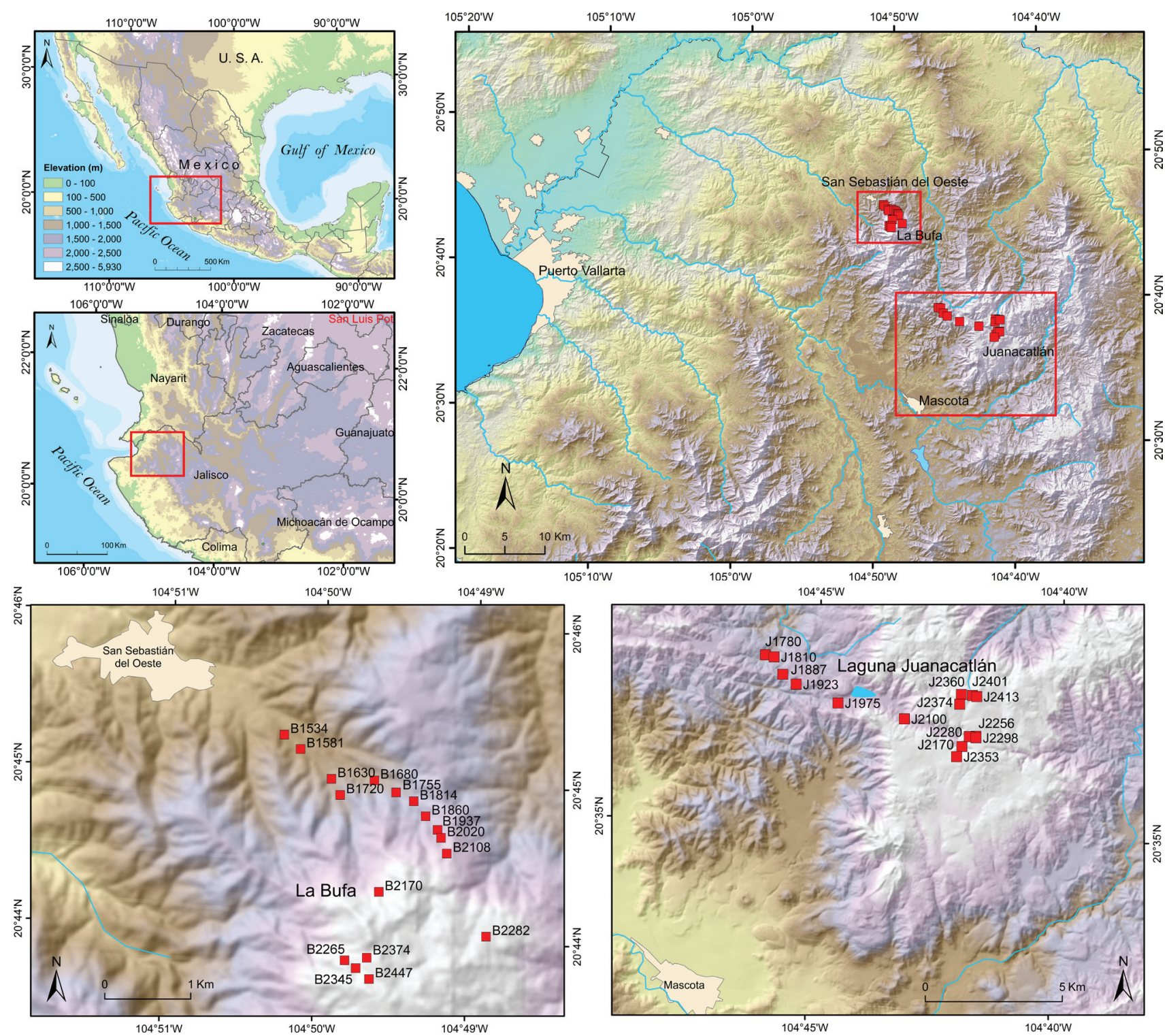

Figura 1. Localización del área de estudio. Dos localidades de estudio, La Bufa y Laguna Juanacatlán. Cuadros con relleno rojo son los sitios de estudio, etiquetados con letra B (Bufa) o J (Laguna Juanacatlán) y un número correspondiente a su altitud en metros.

afloramientos ígneos intrusivos de la Sierra Madre del Sur (Gómez-Tuena et al. 2005). De acuerdo con las divisiones florísticas de México propuestas por Rzedowski (1978), la zona se localiza en la provincia de las Serranías Meridionales y éstas a su vez en la Región Mesoamericana de Montaña. El clima, de acuerdo a la clasificación de Köppen modificada por García (2004), es templado subhúmedo con verano fresco y largo, con lluvias en verano con un porcentaje de lluvia invernal $<5 \%$, el más húmedo de los subhúmedos [C $\left.\left(\mathrm{w}_{2}\right)(\mathrm{w})\right]$. Tiene una temperatura media anual que va de los $18{ }^{\circ} \mathrm{C}$, en las partes más bajas a $13.2^{\circ} \mathrm{C}$ en las zonas con mayor altitud; y una precipitación anual de 1,183 a 1,256 mm (Hijmans et al. 2005).
Diseño de muestreo. Se realizó un muestreo dirigido a los BMM y a los bosques de Abies mejor conservados, sin disturbios antrópicos evidentes, mediante recorridos de campos en dos localidades. El método fue el utilizado por Vázquez-García \& Givnish (1998), con unidades de muestreo de 0.1 ha, las cuales se ubicaron de la siguiente manera: en 32 parcelas de $60 \times 48 \mathrm{~m}$, cada una dividida a su vez en 20 subparcelas de $12 \times 12 \mathrm{~m}$, se seleccionaron de manera estratificada y aleatoria 10 de éstas ( 5 a cada lado de una línea que dividió la parcela mayor en 2 partes de $24 \times 60 \mathrm{~m}$ ). En el centro de cada una de las 10 subparcelas seleccionadas se colocó una estaca, a partir de la cual se trazó un círculo de $5.64 \mathrm{~m}$ de radio para obtener un área de $100 \mathrm{~m}^{2}$ por subparcela, y 
en su conjunto de 0.1 ha por unidad de muestreo. En cada círculo de $100 \mathrm{~m}^{2}$ se identificó, registró y midió el diámetro a la altura del pecho (DAP) y la altura, de cada uno de los individuos de las especies leñosas con diámetro $\geq 2.5 \mathrm{~cm}$. Adicionalmente, en cada uno de los 32 sitios se registraron datos de variables edáficas, geográficas y topográficas. Las variables geográficas y topográficas recopiladas fueron coordenadas de latitud y longitud, altitud, exposición, pendiente
(Tabla 1) y posición topográfica. Se eligieron al menos tres sitios de muestreo por cada intervalo altitudinal de $200 \mathrm{~m}$ de amplitud; al final, el total de unidades de muestreo fue de 17 en La Bufa y 15 en Laguna Juanacatlán. Los ejemplares no identificados en campo se colectaron para ser determinados y herborizados en el herbario IBUG. Esta metodología también ha sido usada en varios estudios de rangos altitudinales de la vegetación, estructura de BMM y bosques de Abies del occi-

Tabla 1. Características de los sitios de estudio a lo largo de dos gradientes altitudinales en el occidente de Jalisco, México

\begin{tabular}{|c|c|c|c|c|c|c|c|c|c|}
\hline Sitio & N Latitud & W Longitud & Alt (m snm) & Pend $\left({ }^{\circ}\right)$ & $\operatorname{Exp}\left({ }^{\circ}\right)$ & $\mathbf{P}_{\mathrm{a}}(\mathbf{m m})$ & T m anual $\left({ }^{\circ}\right)$ & $\mathbf{P}_{\mathrm{TS}}(\mathbf{m m})$ & $P_{\mathrm{TF}}(\mathbf{m m})$ \\
\hline \multicolumn{10}{|c|}{ La Bufa } \\
\hline 1 & $20^{\circ} 45^{\prime} 15.6^{\prime \prime}$ & $104^{\circ} 50^{\prime} 14.8^{\prime \prime}$ & 1534 & 16 & 314 & 1256 & 18 & 29 & 64 \\
\hline 2 & $20^{\circ} 45^{\prime} 10.4^{\prime \prime}$ & $104^{\circ} 50^{\prime} 8.2^{\prime \prime}$ & 1581 & 22 & 294 & 1256 & 18 & 29 & 64 \\
\hline 3 & $20^{\circ} 44^{\prime} 59.6^{\prime \prime}$ & $104^{\circ} 49^{\prime} 55.6^{\prime \prime}$ & 1630 & 25 & 298 & 1228 & 17.1 & 30 & 73 \\
\hline 4 & $20^{\circ} 44^{\prime} 59.9^{\prime \prime}$ & $104^{\circ} 49^{\prime} 38.7^{\prime \prime}$ & 1680 & 14 & 293 & 1228 & 17.1 & 30 & 73 \\
\hline 5 & $20^{\circ} 44^{\prime} 53.6^{\prime \prime}$ & $104^{\circ} 49^{\prime} 51.9^{\prime \prime}$ & 1720 & 34 & 270 & 1228 & 16.6 & 30 & 73 \\
\hline 6 & $20^{\circ} 44^{\prime} 55.7^{\prime \prime}$ & $104^{\circ} 49^{\prime} 30^{\prime \prime}$ & 1755 & 25 & 327 & 1228 & 16.6 & 29 & 73 \\
\hline 7 & $20^{\circ} 44^{\prime} 52.7^{\prime \prime}$ & $104^{\circ} 49^{\prime} 22.9^{\prime \prime}$ & 1814 & 15.5 & 285 & 1215 & 15.1 & 29 & 79 \\
\hline 8 & $20^{\circ} 44^{\prime} 47.2^{\prime \prime}$ & $104^{\circ} 49^{\prime} 18^{\prime \prime}$ & 1860 & 35 & 315 & 1215 & 15.1 & 29 & 79 \\
\hline 9 & $20^{\circ} 44^{\prime} 42.2^{\prime \prime}$ & $104^{\circ} 49^{\prime} 13.1^{\prime \prime}$ & 1937 & 19 & 315 & 1215 & 15.1 & 29 & 79 \\
\hline 10 & $20^{\circ} 44^{\prime} 39.2^{\prime \prime}$ & $104^{\circ} 49^{\prime} 11.6^{\prime \prime}$ & 2020 & 33 & 315 & 1215 & 15.1 & 29 & 79 \\
\hline 11 & $20^{\circ} 44^{\prime} 33.3^{\prime \prime}$ & $104^{\circ} 49^{\prime} 9.1^{\prime \prime}$ & 2108 & 29 & 315 & 1215 & 15.1 & 29 & 79 \\
\hline 12 & $20^{\circ} 44^{\prime} 17.2^{\prime \prime}$ & $104^{\circ} 49^{\prime} 35.2^{\prime \prime}$ & 2170 & 32 & 270 & 1220 & 14.7 & 29 & 82 \\
\hline 13 & $20^{\circ} 43^{\prime} 50.3^{\prime \prime}$ & $104^{\circ} 49^{\prime} 47.6^{\prime \prime}$ & 2265 & 35 & 358 & 1217 & 13.2 & 32 & 89 \\
\hline 14 & $20^{\circ} 44^{\prime} 2.2^{\prime \prime}$ & $104^{\circ} 48^{\prime} 52.4^{\prime \prime}$ & 2282 & 20 & 10 & 1215 & 14.5 & 29 & 79 \\
\hline 15 & $20^{\circ} 43^{\prime} 47.5^{\prime \prime}$ & $104^{\circ} 49^{\prime} 43.1^{\prime \prime}$ & 2345 & 31 & 341 & 1217 & 13.2 & 32 & 89 \\
\hline 16 & $20^{\circ} 43^{\prime} 51.7^{\prime \prime}$ & $104^{\circ} 49^{\prime} 38.9^{\prime \prime}$ & 2374 & 37 & 315 & 1217 & 13.2 & 32 & 89 \\
\hline 17 & $20^{\circ} 43^{\prime} 43.6^{\prime \prime}$ & $104^{\circ} 49^{\prime} 37.7^{\prime \prime}$ & 2447 & 29 & 329 & 1217 & 13.2 & 32 & 89 \\
\hline \multicolumn{10}{|c|}{ Laguna Juanacatlán } \\
\hline 1 & $20^{\circ} 38$ '24.1" & $104^{\circ} 46^{\prime} 6^{\prime \prime}$ & 1780 & 5 & 20 & 1217 & 16.5 & 29 & 72 \\
\hline 2 & $20^{\circ} 38^{\prime} 22.1^{\prime \prime}$ & $104^{\circ} 45^{\prime} 55.2^{\prime \prime}$ & 1810 & 20 & 270 & 1215 & 16.3 & 29 & 73 \\
\hline 3 & $20^{\circ} 38^{\prime} 1.6^{\prime \prime}$ & $104^{\circ} 45^{\prime} 43.6^{\prime \prime}$ & 1887 & 8 & 22 & 1215 & 16.3 & 29 & 73 \\
\hline 4 & $20^{\circ} 37^{\prime} 50.5^{\prime \prime}$ & $104^{\circ} 45^{\prime} 26.5^{\prime \prime}$ & 1923 & 17 & 296 & 1215 & 16.1 & 29 & 74 \\
\hline 5 & $20^{\circ} 37^{\prime} 30.5^{\prime \prime}$ & $104^{\circ} 44^{\prime} 34^{\prime \prime}$ & 1975 & 22 & 322 & 1211 & 15.7 & 28 & 75 \\
\hline 6 & $20^{\circ} 37^{\prime} 15.5^{\prime \prime}$ & $104^{\circ} 43^{\prime} 11.3^{\prime \prime}$ & 2100 & 28 & 317 & 1200 & 15.1 & 29 & 77 \\
\hline 7 & $20^{\circ} 36^{\prime} 45.8^{\prime \prime}$ & $104^{\circ} 41^{\prime} 59^{\prime \prime}$ & 2170 & 32 & 292 & 1188 & 14.3 & 30 & 80 \\
\hline 8 & $20^{\circ} 36^{\prime} 58.9^{\prime \prime}$ & $104^{\circ} 41^{\prime} 42.3^{\prime \prime}$ & 2256 & 31 & 297 & 1188 & 14.3 & 30 & 80 \\
\hline 9 & $20^{\circ} 36^{\prime} 58.1$ & $104^{\circ} 41^{\prime} 50.4^{\prime \prime}$ & 2280 & 21 & 280 & 1188 & 14.3 & 30 & 80 \\
\hline 10 & $20^{\circ} 36^{\prime} 57.2^{\prime \prime}$ & $104^{\circ} 41^{\prime} 42.1^{\prime \prime}$ & 2298 & 18 & 250 & 1188 & 14.3 & 30 & 80 \\
\hline 11 & $20^{\circ} 36^{\prime} 33.2$ & $104^{\circ} 42^{\prime} 5^{\prime \prime}$ & 2353 & 20 & 280 & 1193 & 14.6 & 30 & 79 \\
\hline 12 & $20^{\circ} 37^{\prime} 48.4^{\prime \prime}$ & $104^{\circ} 42^{\prime} 2.2^{\prime \prime}$ & 2360 & 28 & 280 & 1187 & 13.8 & 30 & 83 \\
\hline 13 & $20^{\circ} 37^{\prime} 36.6$ & $104^{\circ} 42^{\prime} 3.7^{\prime \prime}$ & 2374 & 10 & 160 & 1187 & 13.8 & 30 & 83 \\
\hline 14 & $20^{\circ} 37^{\prime} 48.1^{\prime \prime}$ & $104^{\circ} 41^{\prime} 48.4^{\prime \prime}$ & 2401 & 12 & 225 & 1183 & 13.9 & 30 & 81 \\
\hline 15 & $20^{\circ} 37^{\prime} 46.9^{\prime \prime}$ & $104^{\circ} 41^{\prime} 43.1^{\prime \prime}$ & 2413 & 12 & 100 & 1183 & 13.9 & 30 & 81 \\
\hline
\end{tabular}

Coordenadas geográficas, altitud (Alt), pendiente (Pend), exposición (Exp), precipitación anual $\left(\mathrm{P}_{\mathrm{a}}\right)$, temperatura media anual (T m anual), precipitación del trimestre más seco del año $\left(\mathrm{P}_{\mathrm{TS}}\right)$ y precipitación del trimestre más frío del año $\left(\mathrm{P}_{\mathrm{TF}}^{\mathrm{a}}\right)$. 
dente de México (Vázquez-García et al. 2000, Cuevas-Guzmán 2002, Sánchez-Rodríguez et al. 2003, Sahagún-Godínez 2004, Cuevas-Guzmán et al. 2011). Las variables edáficas se determinaron mediante análisis físicoquímico de una muestra mixta de la capa superior del suelo (a una profundidad de $30 \mathrm{~cm}$ ) por cada sitio de estudio (32 muestras en total). Se determinó la textura del suelo con hidrómetro de Bouyoucos, el pH con un potenciómetro Orion research 301 Analog Bench, la cantidad de materia orgánica de acuerdo al método de Walkley \& Black (1934), la capacidad de intercambio de cationes (con el procedimiento de acetato de amonio), nitrógeno inorgánico, $\mathrm{P}, \mathrm{K}, \mathrm{Ca}, \mathrm{Na}$ y $\mathrm{Mg}$, todos con base en los métodos sugeridos en la NOM-021-SEMARNAT-2000 (SEMARNAT 2002).

Análisis de Datos. La diversidad de Shannon-Wiener y el estimador de riqueza ACE (Abundance Coverage Estimator) se calcularon con el programa EstimateS 9.0 (Colwell 2013). Después, se ajustaron ecuaciones lineales y polinomiales con las variables estructurales, diversidad Shannon y riqueza (variables dependientes) en relación con la altitud (variable independiente). Para evaluar las tendencias en casos con distribución de errores tipo Poisson (conteos de individuos y riqueza de especies) se utilizaron modelos lineales generalizados. El mejor modelo fue seleccionado con el criterio de información de Akaike corregido $\left(\mathrm{AIC}_{\mathrm{c}}\right.$ ) (Burnham \& Anderson 2002) utilizando el programa R project 2.5.1 (R Core Team 2007).

La información climática incluyó 19 variables bioclimáticas descargadas de la base de datos de WorldClim (Hijmans et al. 2005) para cada sitio de estudio. La precipitación del trimestre más seco del año y la precipitación del trimestre más frío del año fueron seleccionadas utilizando análisis de regresión y correlación de Pearson, para evitar colinealidad. Las variables bioclimáticas altamente correlacionadas fueron excluidas del análisis de ordenación. Solo dos variables bioclimáticas más (temperatura media anual y precipitación anual) fueron incluidas en este estudio, ya que las temperaturas y precipitaciones del resto de los trimestres estaban altamente correlacionadas.

Para analizar la relación entre la abundancia de especies leñosas y la ordenación de los sitios con las variables ambientales a lo largo del gradiente altitudinal, se utilizó el Análisis de Escalamiento Multidimensional No Métrico (en inglés Non-metric Multidimensional Scaling Analysis, NMDS) del programa PC-ORD 6 (McCune \& Grace 2002) con 24 variables ambientales. La clasificación de los sitios de muestreo se realizó con un Análisis Jerárquico de Agrupamiento (Cluster Analysis), con una matriz que incluyó 32 sitios y 103 especies. Este análisis fue implementado utilizando la medida de distancia Sørensen (Bray-Curtis) y el método de ligamiento Beta Flexible (beta $=-0.25$ ). Las diferencias en la composición de especies entre los grupos obtenidos del Análisis de Agrupamiento fueron evaluadas estadísticamente mediante el Procedimiento de Permutación Multi-Respuesta (MRPP por sus siglas en inglés) (McCune \& Grace 2002). La prueba estadística $(T)$ describe la separación entre grupos (valores negativos reflejan separaciones mayores) y la probabilidad corregida dentro de un grupo $(\Delta)$. Cuando todas las especies dentro de un grupo son idénticas, $\Delta$ alcanza su valor mayor $(\Delta=1)$. Cuando la heterogeneidad entre grupos iguala el nivel esperado al azar entonces $(\Delta=0)$ y cuando existe mayor heterogeneidad dentro de los grupos que el nivel esperado al azar entonces $(\Delta<0)$. En el Procedimiento de Permutación Multi-Respuesta el valor de $p$ es dado para cada prueba de comparación grupal. Para identificar en forma objetiva $\mathrm{y}$ cuantitativa a las especies indicadoras de cada uno de los grupos del dendrograma del Análisis de Agrupamiento, se corrió el Análisis de Especies Indicadoras (ISA, por sus siglas en inglés) con el método de Tichý \& Chytrý (2006). Estos análisis multivariados se corrieron usando la distancia Sorensen. El Análisis de Especies Indicadoras muestra las especies más exclusivas en cada uno de los grupos detectados en el análisis de agrupamiento, ya que utiliza un valor resultante del producto de la abundancia relativa y la frecuencia relativa de las especies en cada grupo seleccionado previamente. Este análisis arroja el valor indicativo máximo para cada grupo y la significancia estadística utilizando la técnica de Monte Carlo basado en 4,999 aleatorizaciones. Las pruebas estadísticas de ordenación NMDS, clasificación, MRPP e ISA fueron ejecutadas con el programa computacional PC-ORD 6.0 (McCune \& Grace 2002).

\section{Resultados}

Composición florística leñosa y estructura de la vegetación. A lo largo de casi 1,000 m de intervalo altitudinal, en las dos localidades, fueron registrados un total de 3,801 individuos en 32 sitios ( 3.2 ha), los cuales pertenecen a 103 especies (incluyendo seis morfoespecies), 63 géneros y 42 familias (Apéndice 1). Las familias mejor representadas en número de especies fueron: Fagaceae (15), Pinaceae (8) y Asteraceae (6). Se resalta que Quercus (15) y Pinus (7) fueron los géneros con más riqueza, mientras que la mayoría de los géneros tuvieron una especie. Las especies más frecuentes (presentes en la mayoría de los sitios) fueron Abies jaliscana (23 sitios), Arbutus xalapensis (19), Quercus obtusata (16), Clethra hartwegii (12), Quercus castanea (12), Rumfordia floribunda (12), Styrax argenteus (12), Roldana angulifolia (11) y Ternstroemia lineata (11). Estas especies representaron, en conjunto, el $44.4 \%$ del número total de individuos.

La estructura de la vegetación en el gradiente altitudinal de Laguna Juanacatlán mostró una tendencia lineal positiva en área basal, altura máxima y altura media (Tabla 3, Figura 2B, C y E); mientras que la densidad (N), índice de ShannonWiener $\left(\mathrm{H}^{\prime}\right)$ y la riqueza de especies $(\mathrm{S})$, tuvieron un patrón unimodal con un pico en el intervalo de los 2,200-2,300 (N) y a 2,100 m (H' y S) de altitud respectivamente (Figura 2A, D y F). En contraste, La Bufa no tuvo tendencias de cambio significativas en el índice de Shannon-Wiener y área basal (Figura 2C y D). Por otro lado, la densidad disminuyó significativamente con respecto a la altitud mientras que la altura media aumentó. La altura máxima tuvo un patrón unimodal con un pico en el intervalo de 1,900 a 2,100 msnm. (Figura $2 \mathrm{E})$. Por último, la riqueza mostró una disminución monotónica en relación con el rango altitudinal $(p=0.0001)$ (Tabla 
Guerrero-Hernández et al. / Botanical Sciences 97 (3): 301-322. 2019

Tabla 2. Valores resultantes de los análisis fisicoquímicos del suelo de los sitios de estudio a lo largo de dos gradientes altitudinales de bosque mesófilo de montaña y bosque de Abies en el occidente de Jalisco, México

\begin{tabular}{|c|c|c|c|c|c|c|c|c|c|c|}
\hline Sitio & $\begin{array}{c}\text { M.O. } \\
(\%)\end{array}$ & $\begin{array}{c}\text { C.I.C. } \\
\text { (meq/100 g) }\end{array}$ & Textura & pH & $\begin{array}{l}\text { N inorg. } \\
\text { (ppm) }\end{array}$ & $\begin{array}{c}P \\
(\mathbf{p p m})\end{array}$ & $\begin{array}{c}\mathrm{K} \\
(\mathrm{meq} / 100 \mathrm{~g})\end{array}$ & $\begin{array}{c}\mathrm{Na} \\
(\mathrm{meq} / 100 \mathrm{~g})\end{array}$ & $\begin{array}{c}\mathrm{Ca} \\
(\mathrm{meq} / 100 \mathrm{~g})\end{array}$ & $\begin{array}{c}\mathrm{Mg} \\
\text { (meq/100 g) }\end{array}$ \\
\hline \multicolumn{11}{|c|}{ La Bufa } \\
\hline 1 & 12.8 & 21.3 & Areno Francoso & 5.3 & 48 & 4 & 1.3 & 1.5 & 12.5 & 2.9 \\
\hline 2 & 22.4 & 60.3 & Franco arenoso & 5.8 & 126 & 0.5 & 1.0 & 0.0 & 13.6 & 4.3 \\
\hline 3 & 22.4 & 16.7 & Franco arenoso & 7.6 & 47 & 1 & 1.5 & 1.5 & 9.0 & 2.8 \\
\hline 4 & 6.7 & 41.5 & Francoso & 7.9 & 51 & 0.1 & 1.0 & 0.0 & 3.8 & 2.5 \\
\hline 5 & 13.9 & 48 & Franco Arenoso & 7.6 & 211 & 1.2 & 1.5 & 0.2 & 25.4 & 5.3 \\
\hline 6 & 17.9 & 41.9 & Francoso & 7.2 & 37 & 19 & 1.1 & 0.1 & 14.2 & 2.8 \\
\hline 7 & 8.3 & 21.3 & Franco limoso & 6.9 & 37 & 2 & 0.8 & 0.6 & 9.6 & 4.4 \\
\hline 8 & 16.9 & 66.8 & Franco Arenoso & 7.0 & 243 & 1.2 & 1.5 & 0.2 & 34.6 & 4.6 \\
\hline 9 & 7.6 & 46.8 & Franco arenoso & 9.4 & 149 & 2.5 & 1.5 & 0.3 & 15.5 & 1.7 \\
\hline 10 & 12.6 & 50.2 & Areno francoso & 6.7 & 121 & 2.6 & 0.8 & 0.2 & 17.0 & 2.1 \\
\hline 11 & 19.9 & 51.4 & Areno francoso & 7.6 & 79 & 2.2 & 0.7 & 0.1 & 10.0 & 1.7 \\
\hline 12 & 25.5 & 56 & Franco Arenoso & 7.2 & 210 & 25 & 1.4 & 0.2 & 29.7 & 2.7 \\
\hline 13 & 14.7 & 42 & Franco arenoso & 6.7 & 79 & 2.4 & 1.3 & 0.2 & 16.5 & 2.4 \\
\hline 14 & 19.3 & 67.4 & Franco Arenoso & 7.1 & 210 & 1 & 1.9 & 0.2 & 37.2 & 3.1 \\
\hline 15 & 14.5 & 61.6 & Areno Francoso & 6.7 & 103 & 2.3 & 0.7 & 0.1 & 17.9 & 2.1 \\
\hline 16 & 16.3 & 57.5 & Areno Francoso & 7.2 & 185 & 2.2 & 1.1 & 0.1 & 16.1 & 1.7 \\
\hline 17 & 17.6 & 53.6 & Franco arenoso & 6.0 & 112 & 11.1 & 1.3 & 0.2 & 11.3 & 2.3 \\
\hline \multicolumn{11}{|c|}{$\begin{array}{c}\text { Laguna } \\
\text { Juanacatlán }\end{array}$} \\
\hline 1 & 7.9 & 16.7 & Areno francoso & 7.2 & 75 & 3.8 & 0.3 & 0.1 & 5.8 & 0.8 \\
\hline 2 & 2.1 & 18.1 & $\begin{array}{c}\text { Franco arcilloso- } \\
\text { arenoso }\end{array}$ & 7.1 & 140 & 0.6 & 0.6 & 0.2 & 3.2 & 4.9 \\
\hline 3 & 6.8 & 30.4 & Areno francoso & 7.6 & 84 & 2.6 & 2.2 & 0.1 & 3.9 & 1.2 \\
\hline 4 & 4.1 & 18.1 & Franco arenoso & 7.6 & 70 & 7.2 & 0.5 & 0.0 & 7.2 & 1.0 \\
\hline 5 & 25 & 23.3 & Areno francoso & 8.4 & 219 & 8.1 & 0.5 & 0.1 & 8.6 & 1.6 \\
\hline 6 & 13.5 & 37 & Franco arenoso & 7.3 & 350 & 1.3 & 0.9 & 0.4 & 4.3 & 1.5 \\
\hline 7 & 2.6 & 30.5 & Franco arenoso & 6.0 & 187 & 23 & 1.7 & 0.2 & 17.7 & 3.4 \\
\hline 8 & 3.1 & 29.9 & Franco arenoso & 5.7 & 149 & 11 & 1.0 & 0.1 & 5.8 & 2.5 \\
\hline 9 & 2 & 32.4 & Franco arcilloso & 6.4 & 145 & 0.5 & 1.1 & 0.3 & 10.7 & 2.7 \\
\hline 10 & 7.5 & 44 & Franco arenoso & 8.1 & 154 & 1 & 1.1 & 0.2 & 11.2 & 1.6 \\
\hline 11 & 13 & 70.3 & Franco arenoso & 6.8 & 126 & 1 & 1.3 & 0.2 & 37.6 & 4.0 \\
\hline 12 & 23 & 73.7 & Franco arenoso & 6.9 & 149 & 1.4 & 1.3 & 0.0 & 42.3 & 3.7 \\
\hline 13 & 4.1 & 29 & Franco & 5.7 & 149 & 0.8 & 1.7 & 0.3 & 7.7 & 1.6 \\
\hline 14 & 15.7 & 55.8 & Franco arenoso & 6.8 & 173 & 0.8 & 1.0 & 0.4 & 12.3 & 1.8 \\
\hline 15 & 15.3 & 49.9 & Areno francoso & 6.6 & 75 & 1 & 1 & 0.1 & 11 & 2 \\
\hline
\end{tabular}

Abreviaturas: M.O. (materia orgánica), C.I.C. (capacidad de intercambio catiónico), pH (potencial de hidógeno, acidez), N inorg. (nitrógeno inorgánico), $\mathrm{P}$ (fósforo), $\mathrm{K}$ (potasio), $\mathrm{Na}$ (sodio), $\mathrm{Ca}$ (calcio), Mg (Magnesio), ppm (partes por millón), meq (miliequivalentes).

3, Figura 2F). Los resultados de los análisis fisicoquímicos del suelo se muestran en la Tabla 2.

Ordenación, clasificación e interrelaciones clima-microambiente-vegetación. La ordenación indirecta NMDS de los 32 sitios (17 de La Bufa y 15 de Laguna Juanacatlán) con base en la abundancia de especies leñosas, resultó en una solución final de dos dimensiones o ejes (stress final: 16.04, 250 iteraciones) (Figura 3). La proporción de varianza acumulada fue $r^{2}=0.65$ (las proporciones de varianza representadas por 
Tabla 3. Resultados del ajuste de modelos lineales $(\mathrm{lm})$ y modelos lineales generalizados $(\mathrm{glm})$ a las variables de estructura y diversidad de especies leñosas en 17 sitios de un gradiente altitudinal en La Bufa y 15 sitios en Laguna Juanacatlán, en función de la altitud. AICc $=$ Criterio de Información Akaike corregido.

\begin{tabular}{|c|c|c|c|c|c|c|c|}
\hline $\begin{array}{c}\text { Variables de estructura } \\
\text { y diversidad }\end{array}$ & $\begin{array}{l}\text { Modelo mínimo } \\
\text { adecuado }\end{array}$ & $\mathrm{AICc}$ & $\begin{array}{c}\text { Intercepto } \\
\text { (a) }\end{array}$ & $\begin{array}{l}\text { Pendiente } \\
\text { (b) }\end{array}$ & $\begin{array}{l}\text { Constante cuadrática } \\
\text { (c) }\end{array}$ & $r^{2} \circ \chi^{2}$ & Valor de $p$ \\
\hline \multicolumn{8}{|l|}{ LA BUFA } \\
\hline DENSIDAD & Lineal $\mathrm{lm}$ & $178.4<180.0$ & 273.46 & -0.07925 & & 0.29 & $0.0262 *$ \\
\hline ÁREA BASAL & Lineal $\mathrm{lm}$ & $69.3<72.6$ & 3.15 & 0.00077 & & 0.02 & 0.5585 n.s. \\
\hline ALTURA MEDIA & Lineal $\mathrm{lm}$ & $81.8<84.4$ & 1.14 & 0.00429 & & 0.26 & $0.0367^{*}$ \\
\hline ALTURA MÁXIMA & $\begin{array}{l}\text { Polinomial } \\
\text { cuadrático lm }\end{array}$ & $124.3<125.9$ & 219.2 & 0.2479 & 0.000059 & 0.38 & $0.0341^{*}$ \\
\hline RIQUEZA (S) & $\begin{array}{l}\text { Lineal glm } \\
\text { (dist. Poisson) }\end{array}$ & $91.9<94.7$ & 4.35 & -0.00081 & & $\chi^{2}=9.07$ & $0.0001 * * *$ \\
\hline DIVERSIDAD $\left(\mathrm{H}^{\prime}\right)$ & Lineal $\mathrm{lm}$ & $18.9<21.5$ & 3.26 & -0.00057 & & 0.2 & 0.0725 n.s. \\
\hline \multicolumn{8}{|l|}{ JUANACATLÁN } \\
\hline DENSIDAD & $\begin{array}{c}\text { Polinomial } \\
\text { cuadrático glm } \\
\text { (dist. Poisson) }\end{array}$ & $274.9<331.2$ & -23.2 & 0.02571 & -0.0000051 & $\chi^{2}=168.5$ & $<0.0001 * * *$ \\
\hline ÁREA BASAL & Lineal $\operatorname{lm}$ & $54.2<57.7$ & -12.1 & 0.00812 & & 0.72 & $<0.0001^{* * *}$ \\
\hline ALTURA MEDIA & Lineal $\mathrm{lm}$ & $64.2<67.1$ & -0.55 & 0.00518 & & 0.35 & $0.02128 *$ \\
\hline ALTURA MÁXIMA & Lineal $\mathrm{lm}$ & $118.9<122.1$ & -46.1 & 0.0404 & & 0.45 & $0.0058^{* *}$ \\
\hline RIQUEZA (S) & $\begin{array}{l}\text { Polinomial } \\
\text { cuadrático glm } \\
\text { (dist. Poisson) }\end{array}$ & $78.7<81.2$ & -23.1 & 0.024 & -0.0000055 & $\chi^{2}=5.82$ & $0.01714 *$ \\
\hline DIVERSIDAD $\left(\mathrm{H}^{\prime}\right)$ & $\begin{array}{c}\text { Polinomial } \\
\text { cuadrático } \mathrm{lm}\end{array}$ & $4.4<14.4$ & -29.4 & -0.0299 & -0.000007 & 0.6 & $0.0039 * *$ \\
\hline
\end{tabular}

el eje 1 y el eje 2 , fueron 0.41 y 0.24 respectivamente). La prueba de Monte Carlo indicó que la probabilidad de que un estrés final similar pudiera haber sido obtenido al azar fue de $p=0.004$.

Las variables ambientales y climáticas que obtuvieron mayor relación con alguno de los dos ejes fueron, para el eje uno: altitud $\left(r^{2}=0.64\right)$, precipitación anual $\left(r^{2}=0.67\right)$, temperatura media anual $\left(r^{2}=0.45\right)$, pedregosidad $\left(r^{2}=0.39\right)$ y precipitación del trimestre más frío $\left(r^{2}=0.28\right)$. Para el eje dos: pendiente topográfica $\left(r^{2}=0.33\right)$, temperatura media anual $\left(r^{2}=0.21\right)$, capacidad de intercambio catiónico del suelo $\left(r^{2}=0.40\right)$, contenido de calcio del suelo $\left(r^{2}=\right.$ $0.43)$, y de los cationes $\mathrm{K}\left(r^{2}=0.21\right)$ y $\mathrm{Ca}\left(r^{2}=0.44\right)$ (Figura 3).

El NMDS separó las parcelas localizadas en altitudes menores de aquellas localizadas en altitudes mayores a lo largo del eje 1 (Figuras 3A, B). Con valores más negativos para el eje 1 se encuentran los sitios de más baja altitud, los cuales están dominados por Carpinus caroliniana, Magnolia pacifica y Quercus centenaria; y con valores positivos se distribuyen los sitios de mayor altitud, dominados por Abies jaliscana, Clethra hartwegii y Q. obtusata. Además, a lo largo del eje 2 los ensambles fueron separados en: bosques dominados por encino-pino (sitios J1780, J1810, J1887, J1975 y B2108), bosques mixtos de alta montaña (sitios J1923, B2170, J2170, B2265, B2345, J2353, J2360 y B2447) y bosques dominados por Abies (sitios J2100, J2256, J2280, B2282, J2298, B2374, J2374, J2401 y J2413) inmersos entre los bosques mixtos de alta montaña. La presencia de cinco grupos de comunidades fue confirmada con su segregación a lo largo de rangos altitudinales, con un Análisis de Agrupamiento Jerárquico Aglomerativo (Hierarchical Agglomerative Cluster Analysis) (Figura 4). El Análisis de Agrupamiento resultó en un dendrograma en el que se distinguen los cinco grupos al realizar un corte vertical al nivel de una información restante del $35 \%$. El primer grupo incluye sitios de La Bufa (B) localizados a baja altitud (de los 1,534 $\mathrm{m}$ a los 2,020 $\mathrm{m})$, el segundo grupo incluyó dos sitios de Juanacatlán (J) de baja altitud (de los 1,887 y 1,975 m) y un sitio de B $(2,108$ $\mathrm{m}$ de altitud); el tercer grupo incluye sitios de B de mayor altitud (entre 2,170 y $2,447 \mathrm{~m}$ ) y entre 1,923 y $2,360 \mathrm{~m}$ en $\mathrm{J}$; el cuarto grupo comprende a los dos sitios de $\mathrm{J}$ de más baja altitud (1,780 y 1,810 m); y el quinto grupo comprende a los sitios restantes de $\mathrm{J}$ de mayor altitud $\mathrm{y}$ a dos sitios de B de 2,282 y 2,374 m. Esta agrupación fue corroborada por el Procedimiento de Permutación Multirespuesta (MRPP), el cual proporcionó información máxima para hacer las separaciones de grupos, la homogeneidad dentro de los cinco grupos fue mayor que la esperada al azar $(T=-12.59, \mathrm{~A}=$ $0.22, p=0.00000)$.

El ISA identificó 12 especies como indicadoras significativas de los grupos $(p<0.05$, Tabla 4$)$. Para el grupo 1 

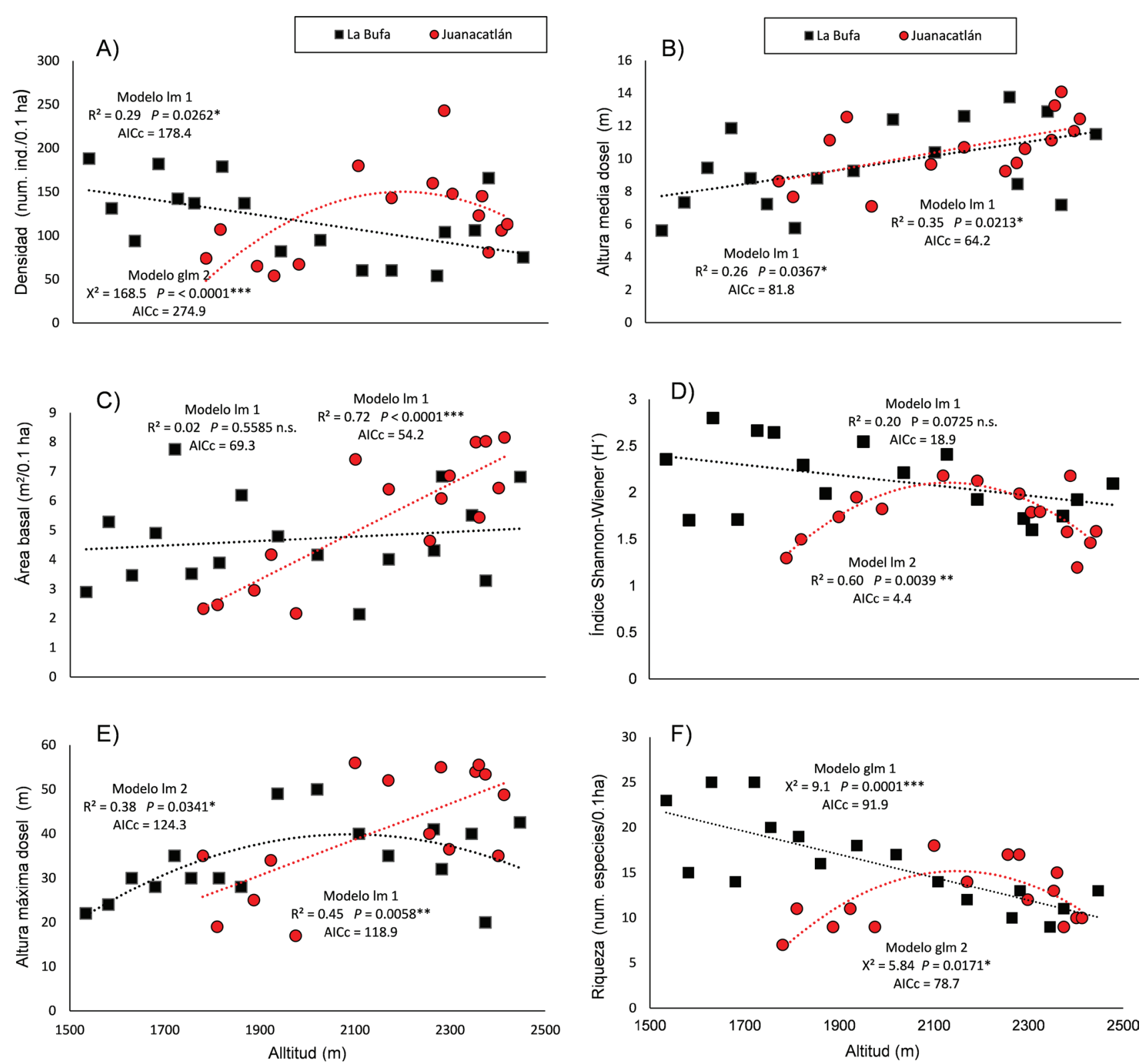

Figura 2. Modelos mínimos adecuados, ajustados a la densidad, área basal, altura promedio, altura máxima, riqueza observada y a los valores del índice de Shannon-Wiener (H') de plantas leñosas en 32 sitios de BMM-bosque de Abies en dos localidades del occidente de México; La Bufa (cuadros) y Laguna Juanacatlán (círculos), en función de la altitud. Modelo lineal (lm), modelo lineal generalizado (glm), modelo 1 (función lineal $\mathrm{y}=\mathrm{a}+\mathrm{bx}$ ), modelo 2 (función Polinomial cuadrática $\left.\mathrm{y}=\mathrm{a}+\mathrm{bx}+\mathrm{cx}^{2}\right)$. $\mathrm{AIC}_{\mathrm{c}}$ (Criterio de Información Akaike corregido).

fueron: Carpinus caroliniana, Clethra fragans, Clusia salvinii, Eugenia culminicola, Magnolia pacifica, Quercus centenaria y Symplococarpon purpusii; para el grupo 2 fueron: Quercus castanea y Rumfordia floribunda; para el grupo 3 fue solamente Clethra hartwegii; para el grupo 4 no hubo especies con valor indicador significativo; y para el grupo 5 las especies indicadoras fueron Abies jaliscana y Quercus obtusata. También resultaron otras 26 especies que tuvieron valor indicador máximo mayor a 0.50 , pero que fueron marginalmente no significativos.
De los cinco grupos discriminados por el análisis de agrupamiento se definieron cuatro tipos de bosque nombrados con base en dos criterios, el primero por sus principales especies dominantes y el segundo por el uso de la nomenclatura de Ohsawa $(1991,1995)$ para bosques de niebla (Tropical Montane Cloud Forests):

Bosque mesófilo de baja montaña (Lower montane cloud forest zone; grupo 1), 1,500-2,020 $\mathrm{m} \mathrm{snm}$. Este es el rango más amplio de bosque, aquí se mezclan especies perennifolias con caducifolias. Los elementos perennifolios como Magno- 
A)

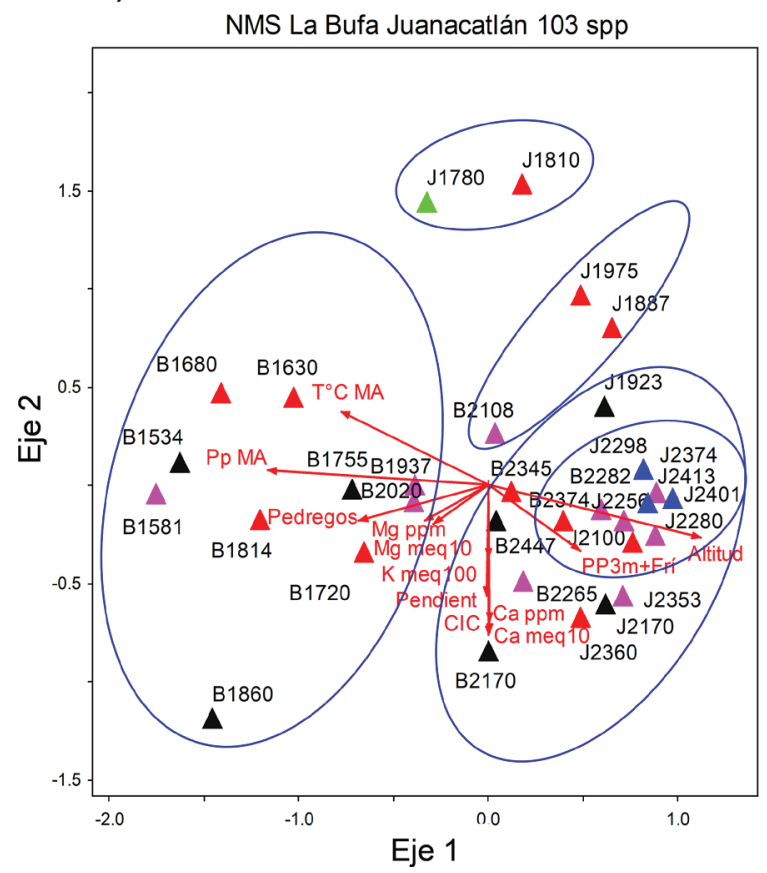

B)

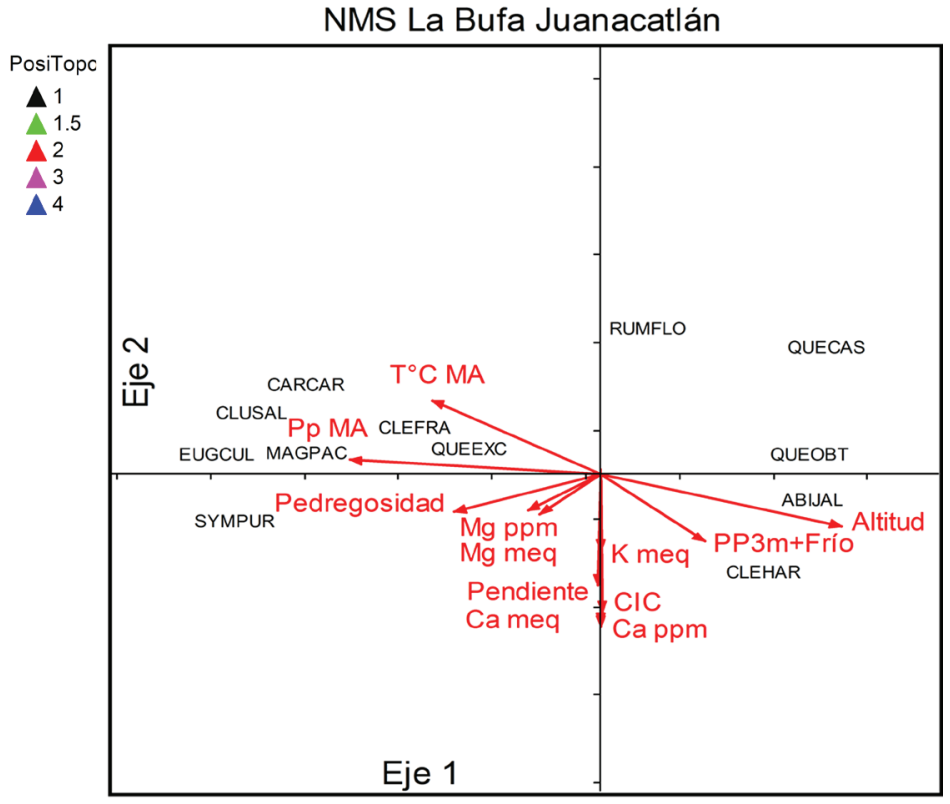

Figura 3. A) Análisis de Escalamiento No Métrico Multidimensional (NMDS) para los 32 sitios y 103 especies leñosas a lo largo del gradiente altitudinal en el occidente de Jalisco, en dos localidades, La Bufa y Laguna Juanacatlán; ( $\mathrm{T}^{\circ} \mathrm{C}$ MA) Temperatura media anual, (Pp) Precipitación anual, (Pp3m+Frío) Precipitación del trimestre más frío del año, (Ca ppm) Contenido de Calcio en el suelo, (Mg ppm) Contenido de Magnesio en el suelo, (Ca meq) Catión de Calcio, (K meq) Catión de Potasio, (Mg meq) Catión de Magnesio. PosiTopo=Posición topográfica: (1) arroyo, (1.5) planicie baja, (2) ladera baja, (3) ladera media y (4) ladera alta. B) NMDS mostrando las 12 especies indicadoras, acrónimos: (ABIJAL) Abies jaliscana; (CARCAR) Carpinus caroliniana; (CLEFRA) Clethra fragans; (CLEHAR) Clethra hartwegii; (CLUSAL) Clusia salvinii; (EUGCUL) Eugenia culminicola; (MAGPAC) Magnolia pacifica; (QUECAS) Quercus castanea; (QUECEN) Quercus centenaria; (QUEOBT) Quercus obtusata; (RUMFLO) Rumfordia floribunda; (SYMPUR) Symplococarpon purpusii.

lia pacifica, Symplococarpon purpusii, Inga hintonii, Clusia salvinii y Symplocos novogaliciana fueron encontrados en el estrato del dosel y subdosel. Las especies caducifolias por su parte fueron representadas principalmente por Carpinus caroliniana, Quercus centenaria, Quercus nixoniana y Ostrya virginiana. Las mayores alturas del dosel fueron dadas por Abies, Carpinus y Magnolia.

Bosque de Quercus-Pinus, 1,780-2,100 m snm. (grupos 2 y 4, parcelas J1,780, J1,810, J1,887, J 1,975, B2,108). Este es un fragmento del gradiente en el que normalmente debería haber BMM. Sin embargo, este bosque es dominado en el dosel por Quercus castanea, $Q$. coccolobifolia, $Q$. martinezii, Q. obtusata, Q. scytophylla y Pinus oocarpa. Entre los elementos mesófilos destacan Alnus acuminata, Oreopanax xalapensis, Ostrya virginiana, Persea hintonii y algunos individuos de Abies jaliscana. Aquí resaltan las especies heliófilas Baccharis salicifolia, Roldana angulifolia y Rumfordia floribundia con una alta abundancia.

Bosque mesófilo de alta montaña (Upper montane cloud forest zone; grupo 3 y una fracción del grupo 5), 1,920-2,450 $\mathrm{m}$ snm. Aquí el dosel superior es dominado ampliamente por Abies jaliscana, el cual alcanza alturas superiores a los 50 metros. A pesar de esto, existe un sotobosque bien desa- rrollado en diferentes estratos que está representado por los géneros Arbutus, Clethra, Cleyera, Ilex, Meliosma, Myrsine, Ostrya, Quercus, Symplocos, Styrax y Ternstroemia.

Bosque de Abies (Coniferous forest zone; una fracción del grupo 5, parcelas J2298, J2374, J2401, J2413). Este grupo fue exclusivamente dominado en todos los estratos por Abies jaliscana, aunque en el sotobosque pueden verse algunos individuos de tallas pequeñas de los géneros Pinus, Prunus, Quercus y Styrax. En esta franja altitudinal la mayor altura del estrato arbóreo fue de Abies, con 53 metros.

\section{Discusión}

En los bosques de las montañas tropicales y subtropicales de diferentes regiones del mundo se han encontrado patrones contrastantes en estructura de la vegetación, riqueza y composición florística a lo largo de gradientes altitudinales (Williams-Linera et al. 2013).

Composición florística leñosa en relación con la altitud. Las familias Fagaceae y Pinaceae fueron dominantes a lo largo de los dos gradientes altitudinales, especialmente en Laguna Juanacatlán, una tendencia similar a los bosques templados 


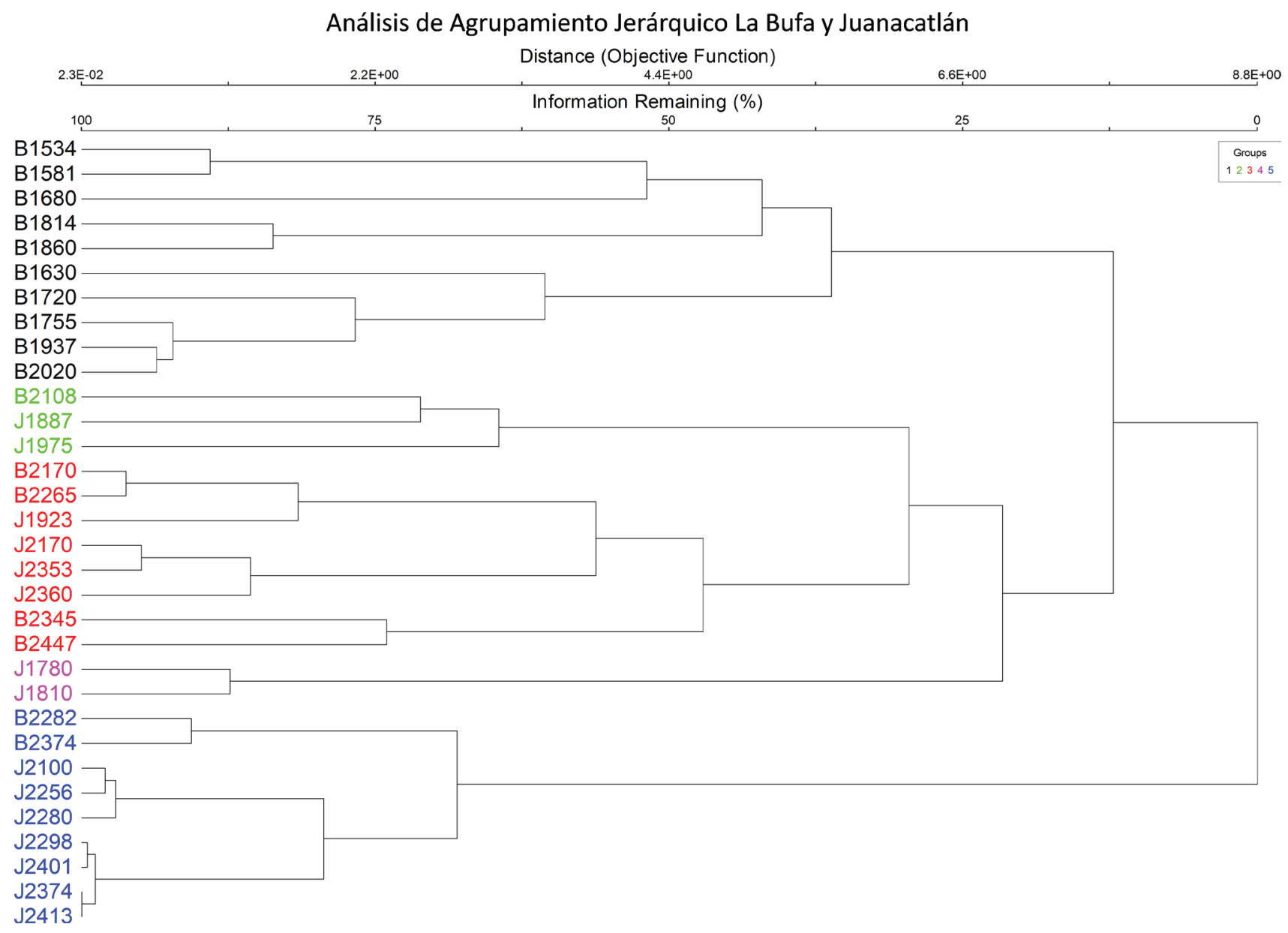

Figura 4. Dendrograma resultante del análisis de agrupamiento de los sitios de estudio entre 1,534 y 2,447 m de altitud en bosque mesófilo de montaña y su transición con el bosque de Abies en el occidente de Jalisco, México. Se distinguen cinco grupos al realizar un corte vertical con una información restante del $35 \%$; La Bufa (B) y Laguna Juanacatlán (J).

en la Sierra Nevada del Estado de México, Sierra de Manantlán y el centro de Veracruz (Sánchez-González \& López-Mata 2003, Olvera-Vargas et al. 2010, Williams-Linera et al. 2013). La riqueza y diversidad de especies leñosas en Laguna Juanacatlán mostró una tendencia unimodal; es decir, aumenta al llegar a elevaciones medias y disminuye en las zonas más bajas y altas, con un pico en los 2,100 y una riqueza mínima en los 1,780 $\mathrm{m}$ de altitud. La tendencia unimodal también ha sido detectada en regiones templadas y tropicales (Tang \& Ohsawa 1997, Sánchez-González \& López-Mata 2003, Williams-Linera et al. 2013). En Cerro Grande, Sierra de Manantlán, aunque la diversidad de especies de plantas vasculares en general disminuye con la altitud, existe una tendencia unimodal en especies arbóreas, pero no es significativa (Vázquez-García \& Givnish 1998). Por lo tanto, nuestros resultados sugieren que la precipitación invernal podría estar relacionada con el patrón unimodal en riqueza de especies leñosas, y estar contribuyendo a la presencia de bosque mesófilo de alta montaña y bosque de Abies; lo cual podría indicar mayor humedad proporcionada por niebla (no medida) en los primeros meses de la temporada seca, que en la zona de bosque de Quercus-Pinus con elementos mesófilos (1,780 m de altitud). En la Bufa hubo una disminución monotónica significativa en riqueza de especies, pero no significativa con el índice de Shannon. El patrón encontrado en La Bufa coincide con lo reportado por otros estudios, en los cuales fue observada una disminución de especies leñosas en las elevaciones más altas (Kitayama 1992, Lieberman et al. 1996, Aiba \& Kitayama 1999, Behera \& Kushwaha 2007, Homeier et al. 2010, Salas-Morales \& Meave 2012). Otros autores han encontrado que la mayor riqueza de especies es explicada por variables relacionadas con alta precipitación y humedad en altitudes intermedias (López-Mata et al. 2012, Williams-Linera et al. 2013). Por el contrario, en la Sierra Madre del Sur, Oaxaca, la temperatura es la variable que explica, en su mayoría, el cambio de riqueza en relación con la altitud (Salas-Morales et al. 2015). La relación precipitación-riqueza de especies en bosques de niebla con coníferas también ha sido estudiada en El Himalaya, donde Abies y Tsuga dominan el rango de altitud más 
Estructura de bosque mesófilo y bosque de Abies

Tabla 4. Especies indicadoras de los cinco grupos determinadas por el Análisis de Especies Indicadoras (ISA)

\begin{tabular}{|c|c|c|c|c|c|}
\hline Especie & VIM & $P$ & Especie & VIM & $P$ \\
\hline \multicolumn{6}{|l|}{ Grupo 1} \\
\hline Carpinus caroliniana & 0.74 & 0.032 & Grupo 2 & & \\
\hline Clethra fragans & 0.87 & 0.000 & Pinus oocarpa & 0.55 & 0.074 \\
\hline Clusia salvinii & 0.67 & 0.024 & Quercus castanea & 0.56 & 0.044 \\
\hline Conostegia volcanilis & 0.51 & 0.071 & Rumfordia floribunda & 0.56 & 0.050 \\
\hline Eugenia culminicola & 0.81 & 0.000 & Grupo 3 & & \\
\hline Eugenia sp. & 0.59 & 0.058 & Clethra hartwegii & 0.56 & 0.001 \\
\hline Euphorbia schlechtendalii & 0.51 & 0.076 & Meliosma dentata & 0.55 & 0.056 \\
\hline Glossostipula concinna & 0.51 & 0.076 & Grupo 4 & & \\
\hline Inga hintonii & 0.59 & 0.060 & Acaciella sp. & 0.67 & 0.059 \\
\hline Magnolia pacifica & 0.74 & 0.032 & Clethra rosei & 0.67 & 0.062 \\
\hline Miconia albicans & 0.41 & 0.510 & Quercus elliptica & 0.56 & 0.131 \\
\hline Parathesis villosa & 0.51 & 0.072 & Quercus magnoliifolia & 0.56 & 0.134 \\
\hline Picramnia guerrerensis & 0.51 & 0.076 & Quercus resinosa & 0.67 & 0.062 \\
\hline Prunus cortapico & 0.51 & 0.080 & Quercus scytophylla & 0.60 & 0.118 \\
\hline Quercus centenaria & 0.76 & 0.000 & Solanum brachystachys & 0.56 & 0.120 \\
\hline Sommera grandis & 0.51 & 0.076 & Vaccinium stenophyllum & 0.667 & 0.0618 \\
\hline Symplococarpon purpusii & 0.87 & 0.000 & Grupo 5 & & \\
\hline Symplocos novogaliciana & 0.51 & 0.072 & Abies jaliscana & 0.34 & 0.045 \\
\hline Synardisia sp. & 0.51 & 0.076 & Quercus obtusata & 0.56 & 0.001 \\
\hline Verbesina fastigiata & 0.51 & 0.079 & & & \\
\hline Vitis sp. & 0.51 & 0.074 & & & \\
\hline Xylosma ciliatifolia & 0.52 & 0.074 & & & \\
\hline Xylosma flexuosa & 0.59 & 0.057 & & & \\
\hline
\end{tabular}

VIM= Valor Indicador Máximo

$P=$ Valor de la probabilidad

elevado y su presencia está positivamente asociada con el incremento de la precipitación anual, humedad relativa y la mayor riqueza de árboles (Wangda \& Ohsawa 2010). Varios autores mencionan que un pico de diversidad en una altitud intermedia a lo largo de gradientes altitudinales podría ser el patron general (Lomolino 2001, Rahbek 2005), mientras que Williams-Linera et al. (2013) señalan que es importante considerar la escala cuando se comparan estudios, para entender los cambios de estructura y la riqueza de especies en el gradiente altitudinal.

Estructura de la vegetación en relación con la altitud. Los estudios sobre cambios estructurales en bosques húmedos a lo largo de gradientes altitudinales han generado resultados diversos. En investigaciones en el centro de Veracruz y La Chinantla, Oaxaca, no se encontraron cambios significativos en área basal y abundancia de árboles con el incremento de la altitud (Rincón 2007, Williams-Linera et al. 2013). En contraste, el gradiente altitudinal de Laguna Juanacatlán mostró incremento de área basal y abundancia en relación con la altitud, lo cual coincide con otras regiones estudiadas
(Vázquez-García \& Givnish 1998, Aiba \& Kitayama 1999, Toledo-Garibaldi \& Williams-Linera 2014). Por otro lado, La Bufa mostró una disminución significativa en abundancia a lo largo del gradiente altitudinal, mientras que en área basal no hubo un cambio significativo, esta última tendencia es similar a otras regiones que no exhiben una disminución o aumento significativo en términos de área basal, como el Monte Emei, China (Tang \& Ohsawa 1997). La tendencia de área basal encontrada en La Bufa quizás se debe a que especies de grandes diámetros (mayores a $1 \mathrm{~m}$ ), prosperan tanto en fajas altitudinales inferiores (Carpinus caroliniana y Magnolia pacifica) como en superiores (Abies jaliscana).

En distintas regiones del mundo se han encontrado varios patrones en la altura del dosel en relación con la altitud. Sin embargo, la tendencia mayormente reportada es que a mayor altitud hay una disminución de la altura máxima y media del dosel (Hamilton et al. 1995, Aiba \& Kitayama 1999, Homeier et al. 2010, Krömer et al. 2013). En contraste, en ambos gradientes del presente estudio fue detectado un incremento significativo de la altura media del dosel a lo 
largo del gradiente altitudinal. El incremento en área basal y altura del dosel en Laguna Juanacatlán podría explicarse por la presencia de bosques mixtos y bosques casi monoespecíficos dominados por Abies jaliscana en altitudes mayores, los cuales presentan diámetros y alturas grandes. En general, la dominancia y presencia de las especies de oyamel o abeto está asociada con alta humedad y baja temperatura a lo largo del año (Rzedowski 1978, Ávila et al. 1994). En el rango altitudinal más elevado del Monte Emei en China, Abies fabri domina tanto el sotobosque como el dosel (Tang \& Ohsawa 1997); sin embargo, este abeto no alcanza las tallas de los abetos mexicanos de la Faja Volcánica Trans-Mexicana, los cuales pueden alcanzar hasta 50 metros de altura (CuevasGuzmán et al. 2011, Guerrero-Hernández et al. 2014). La presencia de coníferas en zonas montañosas templado-frías de México-Centroamérica y el sureste de Asia, es un fenómeno que no ocurre con frecuencia en montañas tropicales, en las cuales se tiende a generalizar que la altura del dosel disminuye monotónicamente en relación con la altitud en los BMM (Hamilton et al. 1995, Scatena et al. 2010).

En el área de estudio, los sitios de mayor altitud mostraron menor precipitación anual que los de menor altitud (Tabla 1). Estos valores difieren de aquellos reportados en la estación climatológica de la Cumbre de Guadalupe (ubicada en la región noroeste de la Sierra de Cacoma, en la Sierra Madre del Sur, $50 \mathrm{~km}$ al sur de Juanacatlán), la cual muestra una precipitación anual de 2,003 $\mathrm{mm}$ a una altitud de $2,120 \mathrm{~m}$ (SMN 2016), en un lugar donde Abies jaliscana también forma comunidades vegetales casi monoespecíficas. Es posible que una menor evapotranspiración y una incidencia constante de niebla (variables no consideradas en WorldClim) son otros factores que podrían jugar un rol en mantener una humedad alta en los sitios en donde Abies es dominante, además de la disminución de la temperatura con el incremento de la altitud.

Ordenación, clasificación e interrelaciones clima-microambiente-vegetación. La altitud es la variable más fuerte en determinar tendencias en distribución y composición de especies leñosas en el BMM, debido a que modula otras variables tales como precipitación, temperatura y humedad. En este estudio, la ordenación NMDS reveló que la pedregosidad, la temperatura media anual más elevada y la mayor precipitación acumulada anual están asociadas con el bosque mesófilo de baja montaña (dominado por Carpinus caroliniana, Magnolia pacifica y Quercus centenaria). El bosque mesófilo de alta montaña y el bosque de Abies estuvieron positivamente asociados con la lluvia de invierno y el incremento altitudinal, lo que confirma la segunda hipótesis de este estudio. En contraste, Toledo-Garibaldi \& WilliamsLinera (2014) encontraron que en el oriente de México el BMM está asociado con la precipitación del trimestre más húmedo del año (PTH) mientras que el bosque de Abies con la altitud. Por otro lado, López-Mata et al. (2012) indicaron, para los bosques húmedos de montaña de México en general, una alta correlación entre riqueza, PTH, altitud, evapotranspiración anual, estacionalidad de la precipitación y carbono orgánico en el suelo.

La distribución de especies leñosas a lo largo de ambos transectos altitudinales apoya la hipótesis individualista de
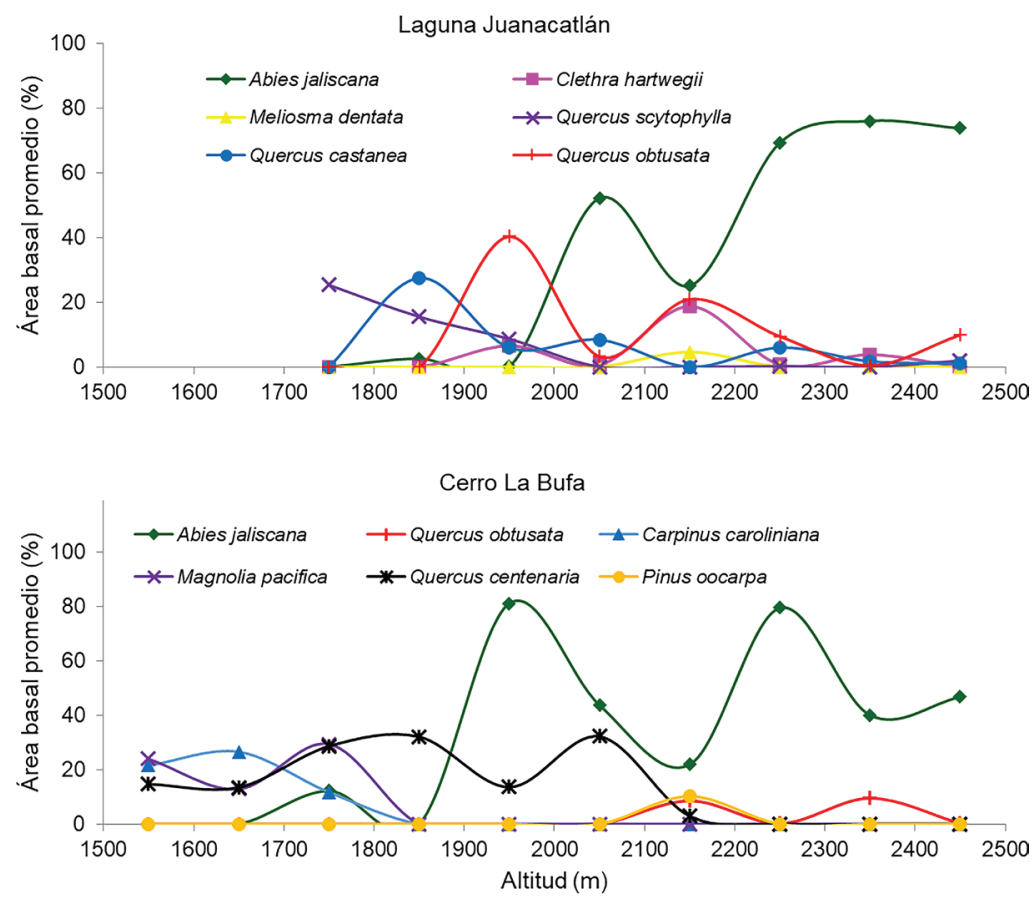

Figura 5. Especies dominantes en área basal, a lo largo de los dos gradientes altitudinales, La Bufa (B) y Laguna Juanacatlán (J). 
Gleason $(1917,1926)$, en la cual se postula que no hay grupos de especies bien definidos y con distribuciones similares, debido a que los centros y límites de la distribución de las especies están dispersos a lo largo de los gradientes altitudinales (Figura 5) (Whittaker 1975). Este enfoque fisonómico, permitió determinar que, para el caso del occidente de México, los límites entre bosque mesófilo de montaña y bosque de Abies no son abruptos, sino continuos, y se traslapan. Varios estudios se han enfocado en analizar solo el gradiente con muestreos en cañadas, crestas, cimas, elevaciones medias y tierras bajas, encontrando diferentes comunidades vegetales, comunidades discretas y discontinuidades (Vázquez-García \& Givnish 1998, Ávila-Sánchez et al. 2010, Hemp 2010). Además, algunos autores han estudiado regiones grandes con condiciones climáticas y ambientales muy diferentes a lo largo de un amplio gradiente altitudinal, como en el centro de Veracruz (Williams-Linera et al. 2013, Toledo-Garibaldi \& Williams-Linera 2014), lo cual explica los cambios un poco más abruptos y la alta heterogeneidad encontrada en los tipos de vegetación.

Los sitios con menor pendiente y menor capacidad de intercambio catiónico en el suelo estuvieron dominados por especies relacionadas con ambientes menos húmedos o con la sucesión temprana, como es el caso de Rumfordia floribunda, Pinus oocarpa, Acaciella sp., Bocconia arborea, Clethra rosei, Quercus magnoliifolia, Quercus coccolobifolia y Quercus resinosa. Estas especies son componentes típicos del bosque de pino-encino y su presencia refleja la influencia tanto de un mayor disturbio antropogénico como de una menor humedad ambiental.

En resumen, a lo largo de los dos gradientes estudiados los patrones de riqueza, área basal y abundancia contrastan en ambas localidades; sin embargo, coinciden en que las mayores alturas de los individuos están dadas por Abies jaliscana. La ordenación y el análisis de agrupamiento, en este estudio, detectaron tres tipos de bosque que forman un continuo y son interpretados como bosque mesófilo de baja montaña, bosque mesófilo de alta montaña y bosque de Abies (desde los 1,500 a los 2,450 m de altitud). El género Abies es casi monodominante, en términos de área basal y abundancia, en cinco parcelas del rango altitudinal de 2,300 a 2,413 m. Los sitios con la menor altitud en Laguna Juanacatlán y un sitio de La Bufa formaron dos grupos distintos, los cuales fueron dominados por Quercus-Pinus. La distribución de estos grupos está ligada principalmente a las diferencias en altitud, precipitación anual e invernal, donde la niebla o precipitación horizontal es importante a considerar para futuros estudios; le siguen en importancia la pedregosidad, algunas variables edáficas y por último, el disturbio con la alta abundancia de especies heliófilas, como Rumfordia floribunda. El contraste de todos estos rasgos con otros bosques mesófilos de montaña de México-Centroamérica y del mundo, sugiere que para definir los cambios en estructura y composición de especies a lo largo de gradientes, se deben considerar todos los elementos con diferentes afinidades biogeográficas que forman zonas de transición o bosques mixtos; por ejemplo, la presencia de Abies y géneros típicos del bosque caducifolio templado como Carpinus, Cornus, Ostrya, Quercus y Tilia, que forman una transición entre el bosque mesófilo de montaña y el bosque de coníferas dominado por Abies.

Finalmente, los resultados indican un avance en la definición de los distintos ensambles en el bosque mesófilo de montaña. El bosque mesófilo de baja montaña, que incluye Carpinus, Conostegia, Inga, Magnolia y Symplococarpon es más diverso que el de alta montaña y el de Abies. El estudio muestra también que distintas especies dominantes están asociadas a diferentes condiciones climáticas y apoya dos tendencias: a) La riqueza de especies en La Bufa, disminuye linealmente con la altitud y se asocia con una mayor precipitación anual y mayor temperatura media anual; b) La riqueza de especies en Laguna Juanacatlán presenta un patrón unimodal, relacionado a una mayor precipitación en invierno; y en los sitios con mayor altitud a una menor temperatura. Estas tendencias apoyan de manera parcial la primera hipótesis de este estudio. La relación del bosque de Abies jaliscana con la precipitación del trimestre más frío (invierno), implica una alta fragilidad climática a la que se encuentra sujeto debido a las fluctuaciones climáticas actuales y pronosticadas a futuro, con posibles cambios en el régimen invernal (inviernos más secos).

En consecuencia, es necesario reforzar políticas de conservación de estos bosques, en especial en la vertiente del Océano Pacífico, donde también son muy heterogéneos (Vázquez-García et al. 2000, Sánchez-Rodríguez et al. 2003, Padilla-Velarde et al. 2008, Guerrero-Hernández et al. 2014), más escasos, discontinuos y relictuales, que los de la vertiente del Golfo de México (Vázquez-García et al. 1995, Vargas-Rodríguez et al. 2010, López-Mata et al. 2012). El realizar estudios acerca de la dinámica de comunidades contribuiría también a su adecuado manejo y conservación, ante la presión antropogénica actual.

\section{Agradecimientos}

Al Consejo Nacional de Ciencia y Tecnología (CONACYT), por la beca 364124 al primer autor.

A la Universidad de Guadalajara, particularmente al posgrado BIMARENA, por apoyos PROINPEP y P3E. Al Dr. Gerardo Hernández Vera y al Dr. Francisco Martín Huerta Martínez, por sus sugerencias para mejorar la calidad del trabajo de investigación. A los Sres. Jaime Arredondo y Modesto Peña por permitir el acceso a sus predios. Agradecemos a dos revisores anónimos, y a los editores Dra. Martha González Elizondo y Dr. Salvador Arias, por sus sugerencias $\mathrm{y}$ críticas que permitieron mejorar el manuscrito final.

\section{Literatura citada}

Aiba SI, Kitayama K. 1999. Structure, composition and species diversity in an altitude-substrate matrix of rain forest tree communities on Mount Kinabalu, Borneo. Plant Ecology 140: 139-157. DOI: https://doi.org/10.1023/A:1009710618040

Alcántara-Ayala O, Luna-Vega I. 1997. Florística y análisis biogeográfico del bosque mesófilo de montaña de Tenango de Doria, Hidalgo, México. Anales del Instituto de Biología de la Universidad Nacional Autónoma de México, Serie Botánica 68: 57-106. 
Alcántara O, Luna I, Velázquez A. 2002. Altitudinal distribution patterns of Mexican cloud forests based upon preferential characteristic genera. Plant Ecology 161: 67-174. DOI: https://doi.org/10.1023/A:1020343410735

Ávila CH, Aguirre JR, García E. 1994. Variación estructural del bosque de oyamel (Abies hickelii Flous y Gaussen) en relación con factores ambientales en el Pico de Orizaba, México. Investigación Agraria. Sistemas y Recursos Forestales 3: 5-17.

Ávila-Sánchez P, Sánchez-González A, Catalán-Everástico C. 2010. Estructura y composición de la vegetación del Cañón del Zopilote, Guerrero, México. Revista Chapingo. Serie ciencias forestales y del ambiente 16: 119-138.

DOI: http://dx.doi.org/10.5154/r.rchscfa.2010.02.003

Bach K, Gradstein RS. 2011. A comparison of six methods to detect altitudinal belts of vegetation in tropical mountains. Ecotropica 17: 1-13.

Behera MD, Kushwaha SPS. 2007. An analysis of altitudinal behavior of tree species in Subansiri district, Eastern Himalaya. Biodiversity and Conservation 16: 1851-1865. DOI: https://doi.org/10.1007/s10531-006-9083-0

Bruijnzeel LA, Scatena FN, Hamilton LS. 2010. Tropical montane cloud forests: science for conservation and management. Cambridge University Press. ISBN-13: 978-0521760355 ISBN-10: 0521760356.

Burnham KP, Anderson DR. 2002. Model selection and multimodel inference: a practical information-theoretic approach. Springer Science \& Business Media. ISBN 978-0-38722456-5

Catalán-Heverástico C, López-Mata L, Terrazas T. 2003. Estructura, composición florística y diversidad de especies leñosas de un bosque mesófilo de montaña de Guerrero, México. Anales Instituto de Biología de la Universidad Nacional Autónoma de México, Serie Botánica 74: 209-230.

Colwell R. 2013. EstimateS: Statistical estimation of species richness and shared species for samples. Version 9.0. < viceroy.eeb.uconn.edu/EstimateS $>$ (accesed August 29, 2013).

CONABIO. 2010. El bosque mesófilo de montaña en México: amenazas y oportunidades para su conservación y manejo sostenible. México, DF: Comisión Nacional para el Conocimiento y Uso de la Biodiversidad. ISBN: 978-607-760735-9

Cuevas-Guzmán R, Benz B, Jardel E, Herrera-MacBryde O. 1997. Sierra de Manantlán región and Biosphere Reserve Mexico. In: Davis SD, Heywood VH, Herrera-MacBryde O, Villa-Lobos J, Hamilton AC, eds. Centres of Plant Diversity: a Guide and Strategy for their Conservation. Washington D.C. The World Wide Fund for Nature \& IUCN, pp. 148152. ISBN: 2-8317-0199-6

Cuevas-Guzmán R. 2002. Análisis de gradientes de la vegetación de la cañada El Tecolote, en la Sierra de Manantlán, Jalisco. PhD Thesis, Colegio de Posgraduados.

Cuevas-Guzmán R, Cisneros-Lepe EA, Jardel-Peláez EJ, Sánchez-Rodríguez EV, Guzmán-Hernández L, Núñez-López NM, Rodríguez-Guerrero C. 2011. Análisis estructural y de diversidad en los bosques de Abies de Jalisco, México. Revista Mexicana de Biodiversidad 82: 1219-1233. DOI: http://dx.doi.org/10.22201/ib.20078706e.2011.4.741
García E. 2004. Modificaciones al sistema de clasificación climática de Köppen. México DF: Instituto de GeografíaUNAM. ISBN: 970-32-1010-4

Gleason HA. 1917. The structure and development of the plant association. Bulletin of the Torrey Botanical Club 43: 463481. DOI: https://doi.org/10.2307/2479596

Gleason HA. 1926. The individualistic concept of the plant association. Bulletin of the Torrey Botanical Club 53: 7-26. DOI: https://doi.org/10.2307/2479933

Gómez-Tuena A, Orozco-Esquivel MT, Ferrari L. 2005. Petrogénesis ígnea de la Faja Volcánica Transmexicana. Boletín de la Sociedad Geológica Mexicana 3: 227-283. http://dx.doi.org/10.18268/bsgm2005v57n3a2

Graham A. 1999. The Tertiary history of the northern temperate element in the northern Latin America biota. American Journal of Botany 86: 32-38. https://doi.org/10.2307/2656952

Grubb PJ. 1977. Control of forest growth and distribution on wet tropical mountains: with special reference to mineral nutrition. Annual Review of Ecology and Systematics 8: 83-107. DOI: https://doi.org/10.1146/annurev.es.08.110177.000503

Guerrero-Hernández R, González-Gallegos JG, Castro-Castro A. 2014. Análisis florístico de un bosque de Abies y el bosque mesófilo de montaña adyacente en Juanacatlán, Mascota, Jalisco, México. Botanical Sciences 92: 541-562.

DOI: http://dx.doi.org/10.17129/botsci.119

Hamilton LS, Juvik JO, Scatena FN. 1995. Tropical montane cloud forests. New York: Springer-Verlag.

https://doi.org/10.1007/978-1-4612-2500-3; ISBN: 978-14612-7564-0

Hemp A. 2010. Altitudinal zonation and diversity patterns in the forests of Mount Kilimanjaro, Tanzania. In: Bruijnzeel LA, Scatena FN, Hamilton LS, eds. Tropical montane cloud forests: science for conservation and management. Cambridge University Press, pp. 134-141. ISBN-13: 978-0521760355; ISBN-10: 0521760356.

Hijmans RJ, Cameron SE, Parra JL, Jones PG, Jarvis A. 2005. Very high resolution interpolated climate surfaces for global land areas. International Journal of Climatology 25: 19651978. DOI: https://doi.org/10.1002/joc.1276

Homeier J, Breckle SW, Günter S, Rollenbeck RT, Leuschner C. 2010. Tree Diversity, Forest Structure and Productivity along Altitudinal and Topographical Gradients in a Species-Rich Ecuadorian Montane Rain Forest. Biotropica 42: 140-148. DOI: https://doi.org/10.1111/j.1744-7429.2009.00547.x

Jardel-Pelaez E, Cuevas-Guzmán R, Santiago AL, Muñoz ME, Aragón-David J. 1996. Nueva localidad y características de la población de Acer skutchii Rehder en la Sierra de Manantlán, Jalisco, México. Acta Botánica Mexicana 35: 13-24. DOI: https://doi.org/10.21829/abm35.1996.954

Kitayama K. 1992. An altitudinal transect study of the vegetation on Mount Kinabalu, Borneo. Vegetatio 102: 149-171. DOI: https://doi.org/10.1007/BF00044731

Krömer T, Acebey A, Kluge J, Kessler M. 2013. Effects of altitude and climate in determining elevational plant species richness patterns: a case study from Los Tuxtlas, Mexico. FloraMorphology, Distribution, Functional Ecology of Plants 208: 197-210. DOI: https://oi.org/10.1016/j.flora.2013.03.003

Lieberman D, Lieberman M, Peralta R, Hartshorn SG. 1996. 
Tropical forest structure and composition on a large-scale altitudinal gradient in Costa Rica. Journal of Ecology 84: 137-152. DOI: https://doi.org/10.2307/2261350

Lomolino MV. 2001. Elevation gradients of species-density: historical and prospective views. Global Ecology and biogeography 10: 3-13. DOI: https://doi.org/10.1046/j.1466-822x.2001.00229.x

López-Mata L, Villaseñor JL, Cruz-Cárdenas G, Ortiz E, Ortiz-Solorio C. 2012. Predictores ambientales de la riqueza de especies de plantas del bosque húmedo de montaña de México. Botanical Sciences 90: 27-36 DOI: http://dx.doi.org/10.17129/botsci.383.

Martin PH, Fahey TJ, Sherman RE. 2011. Vegetation zonation in a neotropical montane forest: environment, disturbance and ecotones. Biotropica, 43: 533-543.

DOI: https://doi.org/10.1111/j.1744-7429.2010.00735.x

Mastretta-Yanes A, Moreno-Letelier A, Piñero D, Jorgensen TH, Emerson BC. 2015. Biodiversity in the Mexican highlands and the interaction of geology, geography and climate within the Trans-Mexican Volcanic Belt. Journal of Biogeography 42: 1586-1600. DOI: https://doi.org/10.1111/jbi.12546

McCune B, Grace JB. 2002. Analysis of ecological communities. Gleneden Beach: MJM Software Design. ISBN-10: 0972129006; ISBN-13: 978-0972129008

Mejía-Domínguez NR, Meave JA, Ruiz-Jiménez CA. 2004. Análisis estructural de un bosque mesófilo de montaña en el extremo oriental de la Sierra Madre del Sur (Oaxaca) México. Boletín de la Sociedad Botánica de México 74: 13-29. DOI: http://dx.doi.org/10.17129/botsci.1684

Ohsawa M. 1991. Structural comparison of tropical montane rain forests along latitudinal and altitudinal gradients in south and east Asia. Vegetatio 97: 1-10.

DOI: https://doi.org/10.1007/BF00033897

Ohsawa M. 1995. The montane cloud forest and its gradational changes in southeast Asia. In: Hamilton FS, Juvik JO, Scatena FN, eds. Tropical Montane Cloud Forests. New York: Springer-Verlag, pp. 254-265.

DOI: https://doi.org/10.1007/978-1-4612-2500-3; ISBN: 978-1-4612-7564-0

Olvera-Vargas M, Figueroa-Rangel BL, Vázquez-López JM. 2010. Is there environmental differentiation in the Quercusdominated forests of west-central Mexico? Plant Ecology 211: 321-335.

DOI: https://doi.org/10.1007/s11258-010-9792-z

Padilla-Velarde E, Cuevas-Guzmán R, Koch SD. 2008. Plantas vasculares y vegetación de la parte alta del arroyo Agua Fría, municipio de Minatitlán, Colima. Acta Botanica Mexicana 84: $25-72$.

R Core Team. 2007. $R$, a language and environment for statistical computing. $R$ Foundation for Statistical Computing, Vienna. <http://www.R-project.org> (accessed November 1, 2015).

Rahbek C. 2005. The role of spatial scale and the perception of large-scale species-richness patterns. Ecology Letters 8: 224239. DOI: https://doi.org/10.1111/j.1461-0248.2004.00701.x

Reich RM, Bonham CD, Aguirre-Bravo C, Chazaro-Basañez M. 2010. Patterns of tree species richness in Jalisco, Mexico: relation to topography, climate and forest structure. Plant
Ecology 210: 67-84.

DOI: https://doi.org/10.1007/s11258-010-9738-5

Rincón A. 2007. Estructura y composición florística de los bosques tropicales húmedos de montaña de Santa Cruz Tepetotutla, Oaxaca, México. MSc. Thesis, Universidad Nacional Autónoma de México.

Rzedowski J. 1978. Vegetación de México. México, DF: Limusa.

Sahagún-Godínez E. 2004. Ordenación del bosque mesófilo en el cerro de La Mona, Sierra de Coalcomán, México, en el contexto del cambio climático. $\mathrm{PhD}$. Thesis. Universidad de Guadalajara.

Salas-Morales SH, Meave JA. 2012. Elevational patterns in the vascular flora of a highly diverse region in southern Mexico. Plant Ecology 213: 1209-1220.

DOI: https://doi.org/10.1007/s11258-012-0077-6

Salas-Morales SH, Meave JA, Trejo I. 2015. The relationship of meteorological patterns with changes in floristic richness along a large elevational gradient in a seasonally dry region of southern Mexico. International Journal of Biometeorology 59: 1861-1874.

DOI: https://doi.org/10.1007/s00484-015-0993-y

Sánchez-González A, López-Mata L. 2003. Clasificación y ordenación de la vegetación del norte de la Sierra Nevada, a lo largo de un gradiente altitudinal. Anales del Instituto de Biología, Universidad Nacional Autónoma de México. Serie Botánica 74: 47-71.

Sánchez-Rodríguez E, López-Mata L, García-Moya E, CuevasGuzmán R. 2003. Estructura, composición florística y diversidad de especies leñosas de un bosque mesófilo de montaña en la sierra de Manantlán, Jalisco. Boletín de la Sociedad Botánica de México 73: 17-34.

DOI: http://dx.doi.org/10.17129/botsci.1676

Scatena FN, Bruijnzeel LA, Bubb P, Das S. 2010. Setting the stage. In: Bruijnzeel LA, Scatena FN, Hamilton LS, eds. Tropical Montane Cloud Forests: science for conservation and management. Cambridge: Cambridge University Press, pp. 3-13. ISBN-13: 978-0521760355 ISBN-10: 0521760356.

SEMARNAT 2002. Norma Oficial Mexicana NOM-021-RECNAT-2000, Especificaciones de Fertilidad, Salinidad y Clasificación de Suelos, Estudio, Muestreo y Análisis. Secretaría del Medio Ambiente y Recursos Naturales. Diario Oficial de la Federación. 2da Sección, 31 de diciembre de 2002.

SMN [Servicio Meteorológico Nacional]. 2016. <http:// smn.cna.gob.mx/es/informacion-climatologica-porestado? estado=jal> (accessed January 15, 2016).

Tang CQ, Ohsawa M. 1997. Zonal transition of evergreen, deciduous and coniferous forests along the altitudinal gradient on a humid subtropical mountain, Mt. Emei, Sichuan, China. Plant Ecology 133: 63-78.

DOI: https://doi.org/10.1023/A:1009729027521

Tichý L, Chitrý M. 2006. Statistical determination of diagnostic species for site groups of unequal size. Journal of Vegetation Science 17: 809-818.

DOI: https://doi.org/10.1111/j.1654-1103.2006.tb02504.x

Toledo-Garibaldi M, Williams-Linera G. 2014. Tree diversity patterns in successive vegetation types along an elevation gradient in the Mountains of Eastern Mexico. Ecological 
Research 29: 1097-1104.

DOI: https://doi.org/10.1007/s11284-014-1196-4

Vargas-Rodríguez YL, Platt WJ, Vázquez-García JA, Boquin G. 2010. Selecting relict montane cloud forests for conservation priorities: the case of western Mexico. Natural Areas Journal 30: 156-173. DOI: https://doi.org/10.3375/043.030.0204

Vázquez-García JA. 1995. Cloud forest archipelagos: Preservation of fragmented montane ecosystems in tropical America. In: Hamilton LS, Juvik JO, Scatena FN, eds. Tropical Montane Cloud Forests. New York: Springer-Verlag, pp. 315-332. DOI: https://doi.org/10.1007/978-1-4612-2500-3; ISBN: 978-1-4612-7564-0

Vázquez-García JA, Givnish TJ. 1998. Altitudinals gradients in tropical forest composition, structure and diversity in the Sierra de Manantlán. Journal of Ecology 86: 999-1020. DOI: https://doi.org/10.1046/j.1365-2745.1998.00325.x

Vázquez-García JA, Vargas YL, Aragón F. 2000. Descubrimiento de un bosque de Acer-Podocarpus-Abies en el municipio de Talpa de Allende, Jalisco, México. Boletín del Instituto de Botánica de la Universidad de Guadalajara 7: 159-183.

Villaseñor JL. 2010. El bosque húmedo de montaña en México y sus plantas vasculares: catálogo florístico-taxonómico.
México, DF.:Comisión Nacional para el Conocimiento y Uso de la Biodiversidad-Universidad Nacional Autónoma de México. ISBN: 978-607-02-1557-5

Walkley A, Black IA. 1934. An examination of the Degtjareff method for determining soil organic matter, and proposed modification of the chromic acid titration method. Soil Science 37: 29-38.

Wangda P, Ohsawa M. 2010. Temperature and humidity as determinants of the transition from dry pine forest to humid cloud forests in the Bhutan Himalaya. In: Bruijnzeel LA, Scatena FN, Hamilton LS, eds. Tropical Montane Cloud Forests: Science for Conservation and Management. Cambridge: Cambridge University Press, pp. 156-163. ISBN-13: 978-0521760355; ISBN-10: 0521760356.

Williams-Linera G, Toledo-Garibaldi M, Gallardo-Hernández C. 2013. How heterogeneous are the cloud forest communities in the mountains of central Veracruz, Mexico? Plant Ecology 214: 685-701.

DOI: https://doi.org/10.1007/s11258-013-0199-5

Whittaker RH. 1975. Communities and Ecosystems. New York: MacMillan. ISBN: 00242739029780024273901

Editor de sección: Martha González-Elizondo

Contribución de los autores: RGH concibió la idea, diseñó el estudio, realizó el trabajo de campo, analizó los datos y escribió el manuscrito; MAMC concibió la idea, diseñó el estudio, consiguió financiamiento, asistió al trabajo de campo y asesoró en análisis de datos; JAVG asesoró en el diseño del estudio y análisis de datos; JARC asesoró en el diseño del estudio y análisis de datos; todos los autores contribuyeron a la discusión, revisión y aprobación del manuscrito. 
Estructura de bosque mesófilo y bosque de Abies

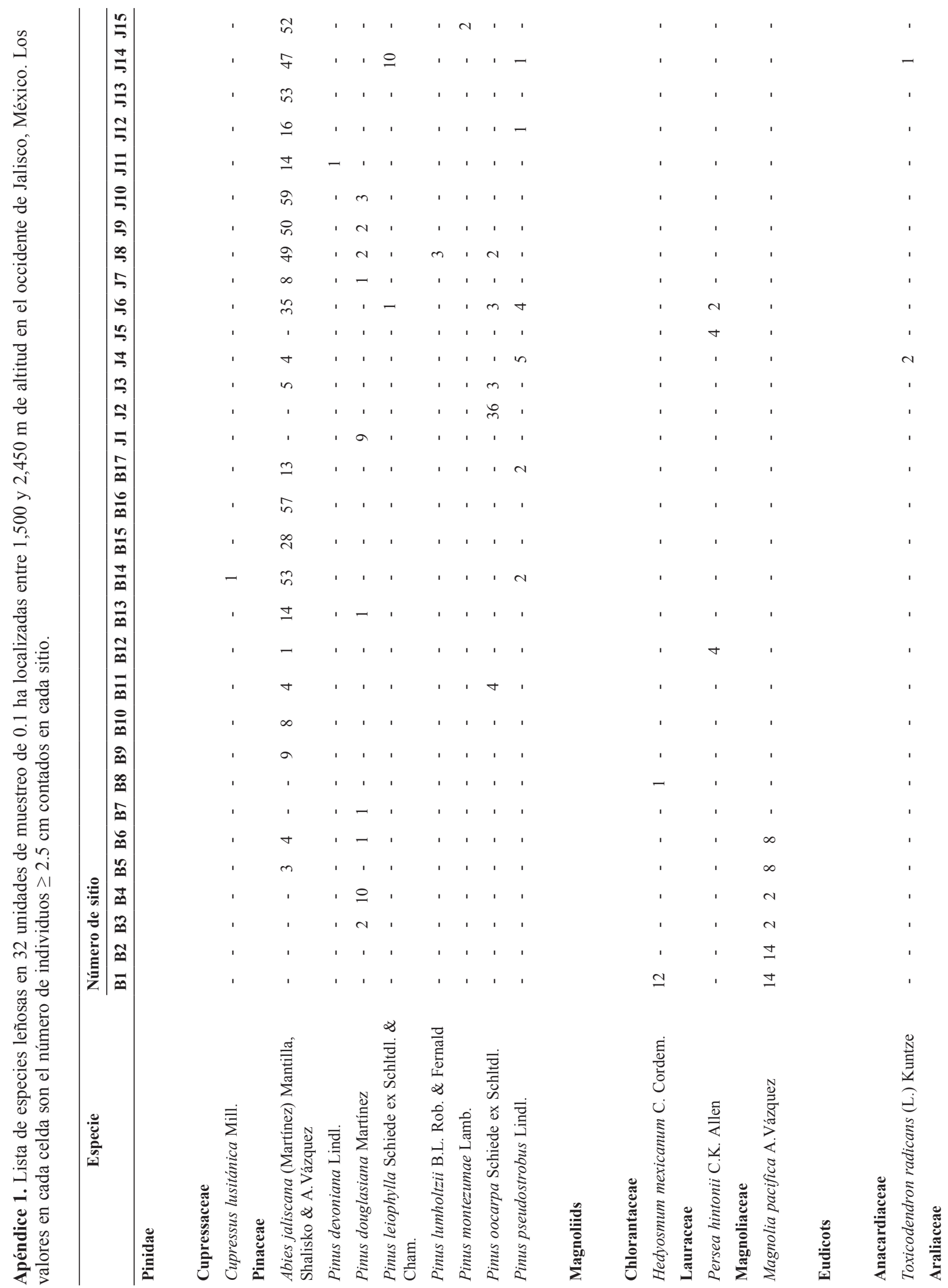


Guerrero-Hernández et al. / Botanical Sciences 97 (3): 301-322. 2019

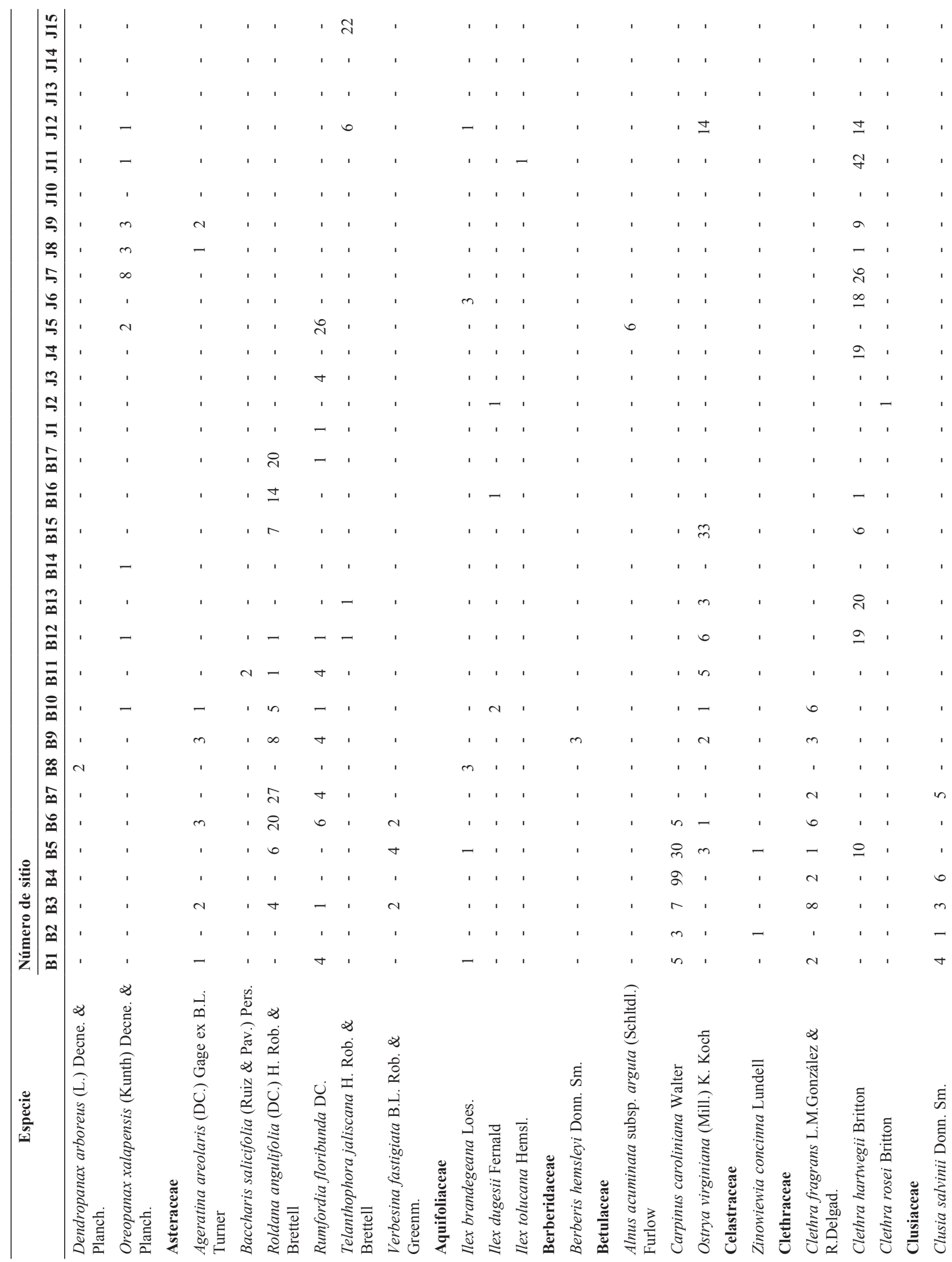


Estructura de bosque mesófilo y bosque de Abies

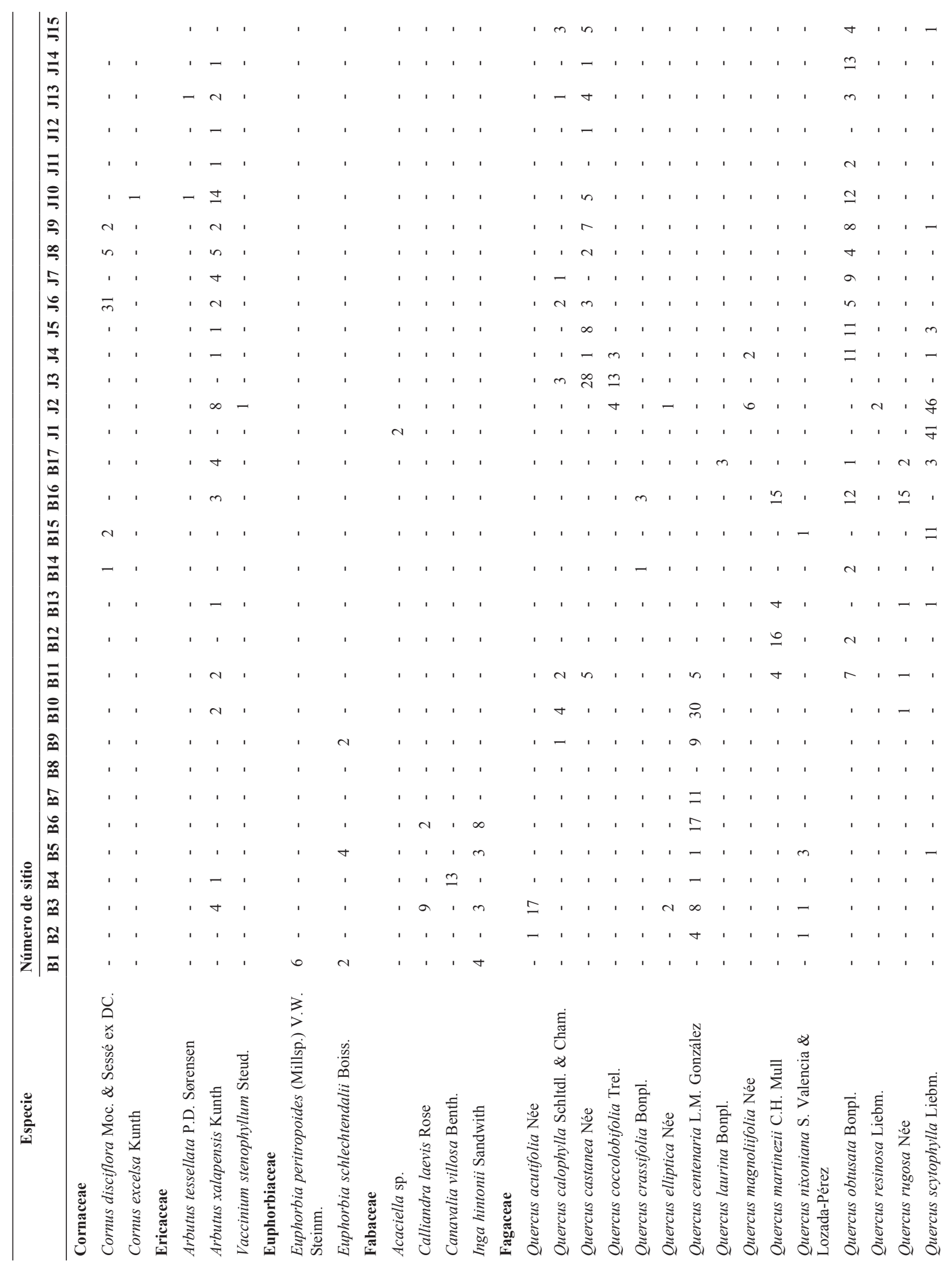


Guerrero-Hernández et al. / Botanical Sciences 97 (3): 301-322. 2019

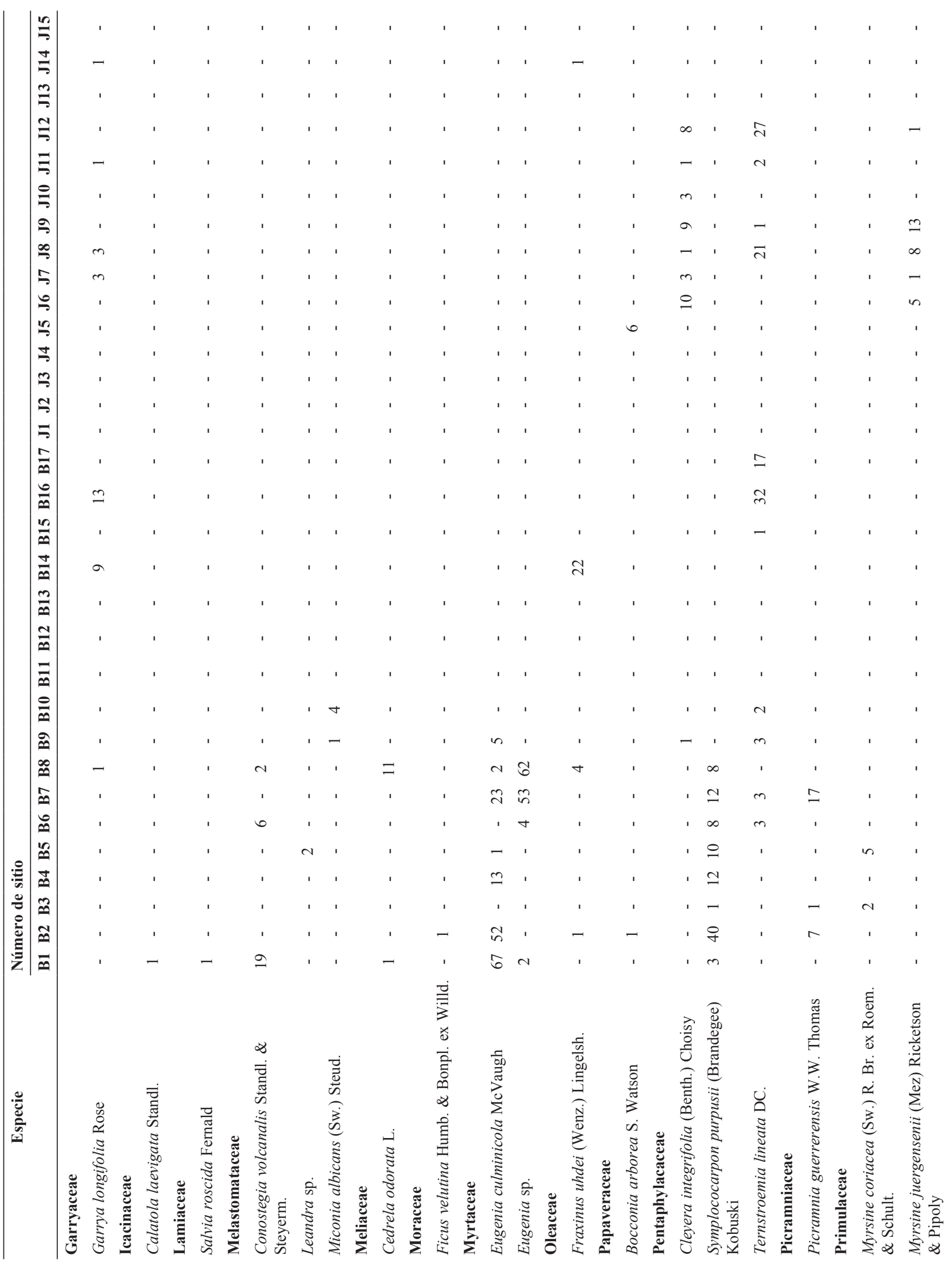


Estructura de bosque mesófilo y bosque de Abies

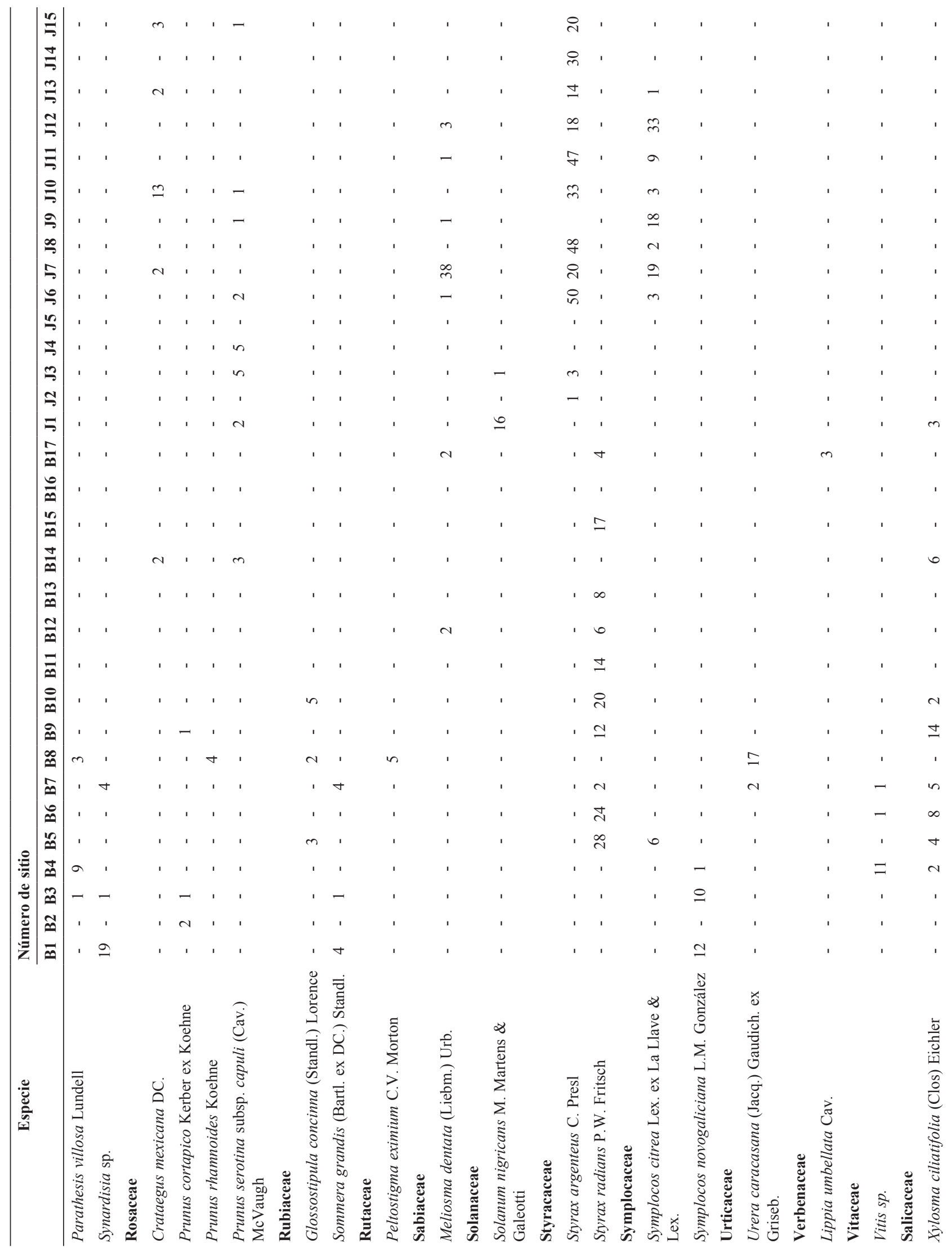


Guerrero-Hernández et al. / Botanical Sciences 97 (3): 301-322. 2019

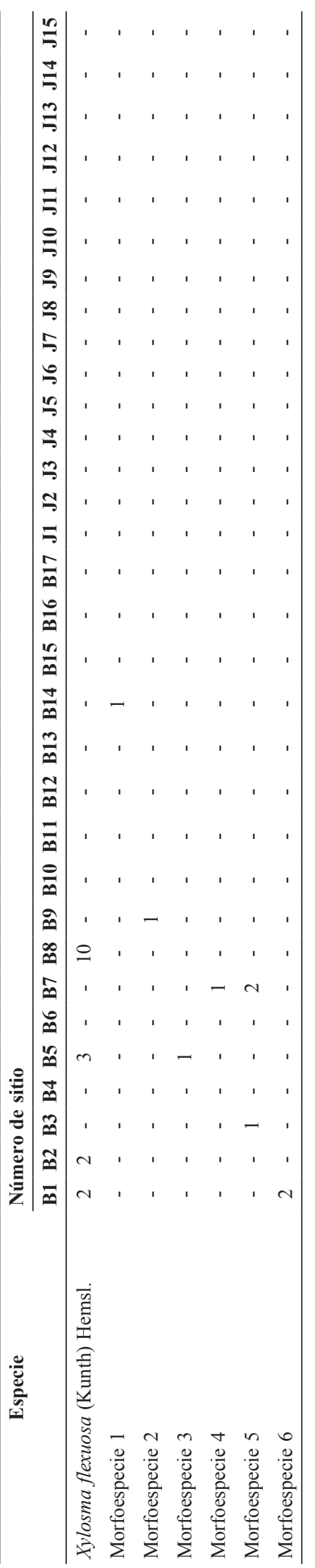

